\title{
South African guideline for management of ischaemic stroke and transient ischaemic attack 2010: A guideline from the South African Stroke Society (SASS) and the SASS Writing Committee
}

\author{
A Bryer, M D Connor, P Haug, B Cheyip, H Staub, B Tipping, W Duim, V Pinkney-Atkinson
}

Background. Stroke is a leading cause of death and disability in South Africa. An increase in the burden of stroke is predicted as the population is undergoing a rapid epidemiological transition with increased exposure to, and development of, stroke risk factors, together with aging of the population.

Objective. The objective was to update the guideline published in 2000, to place the recommendations within the current South African context, and to grade evidence according to the level of scientific rigour.

Recommendations. Ideally, all patients with acute stroke should be managed in a dedicated stroke unit. There is ample evidence that protocol-driven multidisciplinary stroke unit care within a hospital improves recovery from stroke. Treatment in a stroke unit has been shown to reduce mortality as well as reduce the likelihood of dependency after stroke. An effective stroke service requires the establishment of a seamless network consisting of acute stroke units, post-acute care and rehabilitation, and further care in the community.

Primary preventive measures reduce stroke incidence and should be universally available and actively promoted at all levels of health care in South Africa. Successful care of a stroke patient begins with recognition by the public and health professionals that stroke should be considered an emergency. Avoiding delay should be the major aim of the prehospital phase of acute stroke care. Acute stroke or transient ischaemic attack (TIA) should be treated as a medical emergency and evaluated with minimum delay. General supportive treatment is emphasised and is directed at maintaining homeostasis and the treatment of complications. Intravenous thrombolytic therapy with recombinant tissue plasminogen activator (tPA) is an accepted therapy for acute ischaemic stroke within 4.5 hours of onset of symptoms, but can only be administered at centres with specific resources.

Awareness and treatment of the neurological and systemic complications of acute stroke are an integral part of management. Patients with suspected TIA and minor stroke with early spontaneous recovery should be evaluated as soon as possible after an event. Brain imaging is recommended, and non-invasive imaging of the cervicocephalic vessels should be performed urgently and routinely as part of the evaluation. Carotid endarterectomy (CEA) is recommended for patients with severe (70 - 99\%) ipsilateral stenosis, and the procedure should be performed as soon as possible after the last ischaemic event - ideally within 2 weeks - in centres with a peri-operative complication rate (all strokes and death) of less than $6 \%$.

Survivors of a TIA or stroke have an increased risk of another stroke, which is a major source of increased mortality and morbidity. Secondary prevention strategies are aimed at reducing this risk. Stroke rehabilitation is a goal-orientated process that attempts to obtain maximum function in patients who have had strokes and who suffer from a combination of physical, cognitive and language disabilities.

SAfr Med J 2010; 100
Stroke Unit, Groote Schuur Hospital and University of Cape Town

A Bryer, MB BCh, FCP (SA), MMed (Neurology), FC Neurology (SA), PhD

NHS Fife and University of Edinburgh, UK; School of Public Health, University of the Witwatersrand, Johannesburg

M D Connor, MB BCh, FCP (SA), FCNeurol (SA), PhD, FRCP (Edin)

Neurologist in private practice, Cape Town

P Haug, MB ChB, MMed (Int Med), MMed (Neurology), FCP (SA) (Neurology)

Morningside Mediclinic, Sandton

B Cheyip, FCNeurol (SA)

Neurologist in private practice, Life Healthcare Rehabilitation Unit, Entabeni Hospital, Durban

H Staub, MB ChB, FCP (SA) (Neurol)

Division of Geriatric Medicine, Donald Gordon Medical Centre and University of the Witwatersrand

B Tipping, MB ChB, FCP (SA), MPhil, Cert Geriatrics (SA)

Neurologist in private practice, Groenkloof, Pretoria

W Duim, MB ChB, MMed (Neurology), FCP (SA) (Neurol)

Department of Nursing Education, University of the Witwatersrand

V Pinkney-Atkinson, $\mathrm{PhD}, \mathrm{RN}, \mathrm{RM}$

\section{Glossary}

ADL - activities of daily living, AF - atrial fibrillation; BMI body mass index; BP - blood pressure; CAS - carotid angioplasty and stenting; CEA - carotid endarterectomy; CI - confidence interval; CSF - cerebrospinal fluid; CT - computed tomography; CV - cardiovascular; DSA - digital subtraction angiography; DVT - deep-vein thrombosis; DWI - diffusion-weighted imaging; ECG - electrocardiography; EMS - emergency medical services; ESR - erythrocyte sedimentation rate; GCP - good clinical practice; HDL - high-density lipoprotein; HIV - human immunodeficiency virus; INR - international normalised ratio; LDL - low-density lipoprotein; MCA - middle cerebral artery; MRA - magnetic resonance angiography; MRI - magnetic resonance imaging; mRS - modified Rankin score; MDT - multi-disciplinary team; NASCET - North American Symptomatic Carotid Endarterectomy Trial; NG - nasogastric; NIHSS - National Institutes of Health Stroke Scale; NINDS - National Institute of Neurological Disorders and Stroke; NNT - numbers needed to treat; OSA - obstructive sleep apnoea; OR - odds ratio; OT - occupational therapy; PE - pulmonary embolism; PEG - percutaneous enteral gastrostomy; PFO - patent foramen ovale; RCT - randomised controlled trial; RR -relative risk; SASPI - Southern African Stroke Prevention Initiative study; SASS - South African Stroke Society; SSRIs - selective serotonin reuptake 
inhibitors; TCD - transcranial Doppler ultrasound; TIA - transient ischemic attack; tPA - recombinant tissue plasminogen activator.

\section{Objective}

There have been a number of new developments in stroke medicine since the publication of the first South African consensus document - the Stroke Therapy Clinical Guideline 2000. ${ }^{1}$ The benefits of thrombolytic therapy, stroke unit care, new imaging modalities and various other medical and surgical interventions in acute and preventive stroke care have improved. Results of several wellconducted studies now facilitate an evidence-based appraisal of new developments. Numerous evidence-based national guidelines from countries in the developed and developing worlds have been published recently. The new developments have already begun changing stroke management in South Africa. The formulation of an updated South African consensus document was necessary, not only to educate South African health care professionals, students and the general public, but also to guide funding policies of South African private and public health care providers.

This guideline covers several aspects of stroke care, from primary prevention and acute management to rehabilitation and secondary prevention. Stroke care is multifaceted and complex. New evidence emerges every day. A compromise between comprehensiveness and readability needed to be reached, and therefore not all aspects of stroke care could be covered.

\section{Methodology \\ 2.1 Grading the level of evidence}

Most national guideline recommendations either follow the appraisal system used by the American Heart Association, or the definitions of levels of evidence used by the European Stroke Organisation. The validity of both instruments has been well established. This guideline follows the European model.

\section{Evidence appraisal system}

Level A Established as useful/predictive or not useful/ predictive for a diagnostic measure or established as effective, ineffective or harmful for a therapeutic intervention; requires at least one convincing class I study or at least two consistent, convincing class II studies.

Level B Established as useful/predictive or not useful/ predictive for a diagnostic measure or established as effective, ineffective or harmful for a therapeutic intervention; requires at least one convincing class II study or overwhelming class III evidence.

Level C Established as useful/predictive or not useful/ predictive for a diagnostic measure or established as effective, ineffective or harmful for a therapeutic intervention; requires at least two class III studies.

Good Recommended best practice based on the clinical experience of the guideline development group. practice Usually based on class IV evidence indicating large (GCP) clinical uncertainty; such GCP points can be useful for health workers.

\subsection{The guideline development process}

The guideline was compiled by the Stroke Guideline Writing Committee of the South African Stroke Society. This group consists of independent academic and private stroke neurologists and
Evidence classification scheme for a therapeutic intervention

Class I An adequately powered, prospective, randomised, controlled clinical trial with masked outcome assessment in a representative population; or an adequately powered systematic review of prospective, randomised, controlled clinical trials with masked outcome assessment in representative populations.

Class II Prospective matched-group cohort study in a representative population with masked outcome assessment; or a randomised controlled trial in a representative population that lacks one criterion for class I evidence.

Class III All other controlled trials (including well-defined natural history controls or patients serving as own controls) in a representative population, where outcome assessment is independent of patient treatment.

Class IV Evidence from uncontrolled studies, case series, case reports, or expert opinion.

Evidence classification scheme for a diagnostic measure

Class I

A prospective study in a broad spectrum of persons with the suspected condition, using a 'gold standard' for case definition, where the test is applied in a blinded evaluation, and enabling the assessment of appropriate tests of diagnostic accuracy.

Class II A prospective study of a narrow spectrum of persons with the suspected condition, or a welldesigned retrospective study of a broad spectrum of persons with an established condition (by 'gold standard') compared with a broad spectrum of controls, where test is applied in a blinded evaluation, and enabling the assessment of appropriate tests of diagnostic accuracy.

Class III Evidence provided by a retrospective study where either persons with the established condition or controls are of a narrow spectrum, and where test is applied in a blinded evaluation.

Class IV Evidence from uncontrolled studies, case series, case reports, or expert opinion.

physicians. A broader stroke working group provided input to the guideline. Possible conflicts are declared in the attached Register of Interests. Authors were nominated by consensus to write chapters of the Guideline. Submissions were first discussed in a meeting of the Stroke Guideline Writing Committee. The National Department of Health (Directorate Chronic Diseases, Disabilities and Geriatrics) participated in all aspects of the development of the guidelines.

A national consensus meeting of approximately 150 delegates was held on 15 - 17 July 2008; most were from the different regions in the state sector, and the Stroke Society was represented by 15 members. The purpose of the meeting was to discuss the provisional draft of the guidelines and to obtain broad input and consensus on the guidelines from all relevant role players involved in the management and planning of stroke care in the country. The draft document was extensively edited by Associate Professor Alan Bryer 
to include inputs and comments from the consensus meeting as well as further submissions by various role players after reviewing the document. The final draft reflects a broad agreement on appropriate measures for current management of stroke in our unique health care environment.

\subsection{Stroke Writing Committee and Working Group members}

The members of the Stroke Guidelines Writing Committee of the South African Stroke Society are: Chairman and editor: Associate Professor Alan Bryer (Head of the Stroke Unit, Division of Neurology, Department of Medicine, Groote Schuur Hospital and University of Cape Town, chairman of the SA Stroke Society, and stroke portfolio incumbent of the SA Heart and Stroke Foundation); Stroke Guideline Co-ordinator: Dr Victoria Pinkney-Atkinson (SASS); contributing members: Drs Peter Haug (neurologist in private practice, Cape Town), Bonniface Cheyip (neurologist, Witbank Hospital, Mpumalanga Department of Health), Brent Tipping (geriatrician, Donald Gordon Medical Centre, Johannesburg), Hugh Staub (private practice rehabilitation neurologist, Entabeni Hospital, Durban), Wienbren Duim (neurologist in private practice, Little Company of Mary, Pretoria), and Myles Connor (neurologist, Queen Margaret Hospital, Fife, Scotland and University of Edinburgh; formerly of the University of the Witwatersrand). The Stroke Guideline Working Group also included Professor V U Fritz (Emeritus Professor of Neurology, University of the Witwatersrand), Professor A D Marais (Head of Lipidology Division,Department of Medicine, Groote Schuur Hospital and University of Cape Town), Ms Sandhya Singh (Director: Chronic Diseases, Disabilities and Geriatrics, National Department of Health), Dr Marie Strydom (Deputy Director: Geriatrics, National Department of Health), Ms Anne Croasdale (Deputy Director: Chronic Diseases, National Department of Health), Mr Maluta Tshivhase (Deputy Director: Disabilities, National Department of Health), Ms Elmarie van der Walt (Assistant Director: Geriatrics, National Department of Health) and Dr R Cornick (Knowledge Translation Unit, University of Cape Town).

\subsection{Funding of the development and consensus process}

Funding for the planning and convening of the Stroke Guideline Working Group meetings was covered by a grant from Boehringer Ingelheim to the SASS. The grant was unconditional in that the donor made no input to the content of the guideline. The National Department of Health sponsored the consensus meeting held in 2008.

Reference

1. Stroke Therapy Clinical Guideline. S Afr Med J 2000;90:280-306

\section{Stroke in South Africa 3.1 Stroke is a catastrophic illness in South Africa}

Stroke was declared a catastrophic illness in South Africa on 26 October 2007 at a historic ceremony that formed part of the Joint World Congress of Stroke. ${ }^{1}$ This declaration was endorsed by the three organising societies (the International Stroke Society, Mediterranean Stroke Society, and South African Stroke Foundation) as well as the World Stroke Federation. It was intended to focus both South African and world attention on the current burden and future impact of stroke on South Africans. Stroke is largely preventable by reducing vascular risk factors in the population (primary prevention), detecting and effectively managing individuals with stroke risk factors, and preventing stroke recurrence in those who have suffered a stroke through both lifestyle change and medical means (secondary prevention). Notwithstanding the government's achievements to date (in terms of the Tobacco Products Control Amendment Act 63 of 2008), a concerted effort is required by government, health professionals and individuals at risk to address issues relating to vascular risk, including lifestyle modification and treatment of medical conditions that confer increased stroke risk. Should such efforts fail, the burden of stroke in South Africa will increase and add to the burden of disease already facing the nation. ${ }^{2}$

\subsection{Stroke epidemiology in South Africa}

\subsubsection{The current burden of stroke in South Africa}

The assessment of the burden of stroke in a population is based on the number of people who die from stroke (mortality), the number of people in the population at a given time who have survived a stroke (prevalence), and the number who have a stroke during a given year (incidence). We do not know the incidence of stroke in South Africa, but we do have data on stroke mortality and prevalence that highlight the impact of stroke on the population. ${ }^{3}$

\subsubsection{Stroke mortality}

The South African National Burden of Disease Study estimated stroke mortality for the year $2000{ }^{2}$ The finding was that stroke was the third most common cause of death (6.5\% of all deaths) after HIV/ AIDS and ischaemic heart disease in South Africa (age-standardised mortality of stroke for both males and females in 2000 was 125 per 100 000). ${ }^{4}$ Black women had the highest mortality rate owing to stroke (160 per 100000 ), while mortality was lowest in white men (72 per 100 000). Deaths in the coloured and black population groups were double those in the white population. The risk of stroke increases with age, and it is therefore not surprising that there are more stroke deaths in older than in younger age groups in South Africa. According to statistics based on death registration, stroke is the most common cause of death of people over the age of 50 years. ${ }^{5}$ More recent data from StatsSA report that there were just over 25000 deaths from stroke in 2007.6

\subsubsection{Stroke prevalence}

The Southern African Stroke Prevention Initiative study (SASPI, 2004) has provided the only community-based data on the prevalence of stroke in South Africa. The study assessed the prevalence of stroke (number of people in a given population with stroke at any given time) in the Agincourt demographic surveillance site in Mpumalanga. Stroke was about half as common in rural South Africa as in typical high-income populations of the world, but twice that found elsewhere in Africa. Specifically, the age-standardised prevalence of stroke was 290 per 100000 , and the crude prevalence was 300 per 100000 (95\% confidence interval (CI) 250 - 357 per 100 000). ${ }^{7}$ It would be ideal to use these findings to provide an estimate of the total number of people in South Africa who have had a stroke. Unfortunately, there are so many unknown factors that influence this figure (such as population and urban-rural differences in stroke occurrence (incidence), stroke death (mortality) and the proportion of older and younger people) that an estimate based on SASPI findings would be extremely inaccurate. The SASPI study does, however, provide a very useful glimpse of the prevalence of stroke in rural South Africa. Stroke prevalence in urban areas is probably higher than in rural areas because people are probably exposed to more lifestyle risk factors. 


\subsubsection{Stroke-related disability}

The SASPI study compared the prevalence of stroke survivors who required help with at least one activity of daily living (a marker of disability) with similar figures from Tanzania and New Zealand. There were far more disabled stroke survivors in rural South Africa than in Tanzania, and about the same number as in New Zealand, which has a high-income population with (most likely) a higher incidence of stroke than South Africa. ${ }^{7}$ It is not clear why there are so many disabled stroke survivors in South Africa. This finding may be the result of inadequate rehabilitation services, but may also be because minor strokes are not often diagnosed or reflect differences between the research studies in the two countries. Other explanations include: unwillingness by patients to receive rehabilitation or to complete a rehabilitation programme owing to fear of being denied or withdrawn from a disability grant if fully rehabilitated; a lack of transport to attend outpatient rehabilitation sessions for patients travelling long distances to the nearest clinic or hospital; and delays in management of acute stroke.

The South African National Burden of Disease Study estimated national disability-adjusted life years (DALYs) and years of life lost due to premature death. ${ }^{2}$ These are rather complex measures that were first used by the WHO and the Global Burden of Disease Study to compare the level of disability and the years of life that are potentially lost as a result of a disease across populations and countries. Despite the high prevalence of HIV/AIDS and other infections which cause disability, as well as disability due to violence and trauma in South Africa, stroke is the 8th most significant cause of years of life lost due to illness, and the 9th most important cause of disability.

\subsection{Risk factors for stroke}

Risk factors for stroke may be modifiable (i.e. risk that may be avoided or reduced) or non-modifiable (i.e. factors such as increasing age and male gender). A more detailed account of what is known about these risk factors in South Africa is available in a recent Medical Research Council (MRC) technical report. ${ }^{8}$

The South African Comparative Risk Assessment Collaborating Group (2007) recently estimated the contribution of 8 risk factors to stroke. ${ }^{9}$ These are listed in order of their contribution (with attributable fraction in brackets) from highest to lowest:

- high blood pressure (52\%)

- tobacco $(24 \%)$

- excessive body weight (18\%)

- high cholesterol (15\%)

- physical inactivity $(12 \%)$

- low fruit and vegetable intake (12\%)

- diabetes (8\%)

- alcohol (8\%).

These risk factors frequently coexist.

The prevalence of risk factors for stroke varies across the population groups in South Africa, as found in over 9000 people over the age of 30 years without stroke attending general practices, in the South African Stroke Risk in General Practice study. ${ }^{10}$ Hypertension was the most common risk factor in all population groups, and was found in more than half of people attending general practices (55\%), while elevated cholesterol was common in the white (37\%) but not the black (5\%) population groups, while diabetes was most common in the Indian/Asian population groups (24\%).

\subsection{Types of stroke}

There are two main types of stroke:

- ischaemic stroke (85\%), caused by an embolus or thrombosis

- cerebral haemorrhage (15\%), caused by the rupture of a cerebral vessel with bleeding into the brain.
There are also several subtypes of each of these conditions. The relative proportion of the types of stroke varies across populations, probably because of differences in their risk factor profiles, e.g. in populations with a high prevalence of hypertension but low levels of other risk factors, cerebral haemorrhage may be relatively more common than in populations with a greater mix of lifestyle risk factors. ${ }^{8,11}$

\subsection{Prevalence of stroke types in South Africa}

Stroke in South Africa reflects the prevalence and combination of risk factors found in the different population groups of the country. For example, in a recent hospital-based stroke series, cerebral haemorrhage (mainly the result of hypertension) was found twice as often in black $(28 \%)$ as in white $(15 \%)$ stroke patients, ${ }^{11}$ which is typical of the findings from other South African and African hospital-based stroke studies that have found cerebral haemorrhage in around a third of black stroke patients. ${ }^{9}$

Extracranial atherosclerotic disease, a common cause of ischaemic stroke in white and Indian/Asian stroke patients, is uncommon in black stroke patients. ${ }^{12,13}$ Although the incidence of stroke increases with increasing age in the black South African population as in other population groups, some studies have found that the incidence of stroke in younger age groups ( $35-54$ years) is higher than that found in other populations. ${ }^{3}$ Furthermore, stroke management particularly in young South Africans - is complicated by the high prevalence of human immunodeficiency virus (HIV). HIV infection may cause stroke through opportunistic infections, secondary to involvement of the heart by HIV, possibly by changes in coagulation factors, and through direct or indirect damage to blood vessels (HIVassociated vasculopathy). ${ }^{10,14-16}$

\subsection{The cost of stroke}

Stroke carries with it an inherent cost to the affected individual, their family and carers, the community and, more broadly, the health services and country. Unfortunately, there is almost no information available to accurately guide estimation of these costs. ${ }^{17}$

\subsection{The future of stroke in South Africa}

Much of the population of South Africa is undergoing a rapid epidemiological transition with increased exposure to, and development of, stroke risk factors, together with ageing (of the population); $;^{10,18,19}$ this will inevitably result in an increase in the burden of stroke.

\section{Recommendations}

- Educational programmes to increase awareness of stroke at the population level (Class II, Level B)

- Educational programmes to increase stroke awareness among professionals (paramedics, emergency physicians) (Class II, Level B).

\section{References}

1. Culebras A. International Newsletter. Neurology 2006;67:2099-2100.

2. Bradshaw D, Groenewald P, Laubscher R, et al. Initial burden of disease estimates for South Africa, 2000. S Afr Med J 2003;93:682-688.

3. Connor MD, Walker R, Modi G, Warlow CP. Burden of stroke in black populations in sub-Saharan Africa. Lancet Neurology 2007;6:269-278.

4. Norman R, Bradshaw D, Schneider M, Pieterse D, Groenewald P. Revised Burden of Disease Estimates for the Comparative Risk Factor Assessment, South Africa 2000. Cape Town: Medical Research Council, 2006.

5. Statistics South Africa. Mortality and Causes of Death in South Africa 2005: Findings from Death Notification. Statistical Release P0309.3. Pretoria: Statistics South Africa, 2007.

6. Statistics South Africa. Mortality and Causes of Death in South Africa, 2007: Findings from Death Notification. Pretoria: Statistics South Africa, 2009.

7. Connor MD, Thorogood M, Casserly B, Dobson C, Warlow CP, on behalf of the SASPI project team. Prevalence of stroke survivors in rural South Africa: results from the Southern Africa Stroke Prevention Initiative (SASPI) Agincourt field site. Stroke 2004;35:627-632. 
8. Connor M, Bryer A, Steyn K, Fourie J. Chronic Diseases of Lifestyle Technical Report. Tygerberg MRC, 2005.

9. Norman R, Bradshaw D, Schneider M, et al. A comparative risk assessment for South Africa in 2000 towards promoting health and preventing disease. S Afr Med J 2007;8:637-641.

10. Connor M, Rheeder P, Bryer A, et al. The South African stroke risk in general practice study. S Afr Med J 2005;95:334-339.

11. Yusuf S, Reddy S, Ounpuu S, Anand S. Global burden of cardiovascular diseases: part I: general considerations, the epidemiologic transition, risk factors, and impact of urbanization. Circulation 2001;104:2746-2753.

12. Connor MD, Modi G, Warlow CP. Pathological stroke type and ischaemic stroke subtype differs between population groups in urban, hospital-based South African stroke patients: the Johannesburg Hospital Stroke Register. International Journal of Stroke 2006;1:3-46.

13. Fritz VU, Voll CL, Levien LJ. Internal carotid artery occlusion: clinical and therapeutic implications. Stroke 1985;16:940-944.

14. Tipping B, de Villiers $L$ Wainwright $\mathrm{H}$, Candy $\mathrm{S}$, Bryer A Stroke in patients with human immunodeficiency virus infection. J Neurol Neurosurg Psychiatry 2007;78:1320-1324.

15. Modi G, Modi M, Mochan A. Stroke and HIV - causal or coincidental co-occurrence? S Afr Med J 2006;96:1247-1248

16. Connor MD. Stroke in patients with human immunodeficiency virus. J Neurol, Neurosurg Psychiatry 2007;78:1291.

17. Pestana JA, Steyn $\mathrm{K}$, Leiman A, Hartzenberg GM. The direct and indirect costs of cardiovascular disease in South Africa in 1991. S Afr Med J 1996;86:679-684.

18. Vorster HH. The emergence of cardiovascular disease during urbanisation of Africans. Public Health Nutrition 2002;5:239-243.

19. Steyn K, Sliwa K, Hawken S, et al, for the INTERHEART Investigators in Africa. Risk factors associate with myocardial infarction in Africa: The INTERHEART Africa Study. Circulation 2005:112.3554-

\section{The stroke unit model of care \\ 4.1 Organisation of stroke services and benefits of stroke unit care}

- The most effective care for stroke patients is provided in a geographically defined ward area where care is provided by a specialised, experienced stroke team. ${ }^{1-5}$

- The structure of stroke unit care varies between facilities, but all provide care according to protocols, and have regular team meetings and access to ongoing education.

- Treatment in a stroke unit compared with treatment in a routine clinical setting has been shown in studies to reduce mortality as well as reduce the likelihood of dependency after stroke. ${ }^{2}$

- Stroke unit care as provided in routine clinical practice has also been shown to reduce case fatality.,

- All types of stroke patients benefit from treatment and rehabilitation in stroke units: males and females, young and elderly stroke patients, and patients with mild, moderate and severe strokes.

- Stroke unit care incorporates many elements working together, and it is difficult to identify any specific factor responsible for better outcome.

\subsection{Defining a stroke unit}

- A stroke unit is a dedicated and geographically defined part of a hospital that takes care of stroke patients in both the acute and immediate post-acute phase.

- It has specialised staff with a co-ordinated multidisciplinary expert approach to treatment and care.

- It comprises core disciplines: medical, nursing, physiotherapy, occupational therapy, speech and language therapy, and social work. $^{1}$

\subsection{Essential components of a stroke unit}

- Comprehensive assessment of medical problems, impairments and disabilities by specialist staff (i.e. professionals interested and trained in stroke care).

- Established pathways and management protocols for acute and post-acute management of stroke (including pre-hospital and emergency unit management of stroke) with careful attention to active management of physiological abnormalities to maintain homeostasis

- Care co-ordinated by a multi-disciplinary team (MDT) with regular scheduled ward rounds attended by the full MDT to discuss management strategy for each patient. The co-ordinated
MDT should ideally include a stroke physician, nursing staff, occupational therapist, physiotherapist, speech pathologist, dietician, social worker and, where possible, a psychologist.

- Should ideally be in a designated space within hospital, with designated stroke unit beds.

- Early mobilisation.

- Skilled nursing care.

- Early initiation of rehabilitation plan involving the carers

- Scheduled patient and family education concerning management, rehabilitation programme, causes of stroke, secondary prevention and community resources.

- Early assessment and planning of discharge needs and planning for home-based care with either family member, care-giver or community rehabilitation worker.

- Initiation of secondary prevention strategies.

- All staff must undertake ongoing training and education in stroke management.

\section{Recommendations}

- All stroke patients should be treated in a stroke unit (Class I, Level A).

- Health care systems must ensure that acute stroke patients can access high-technology medical and surgical stroke care when required (Class III, Level B).

- The development of clinical networks, including telemedicine, is recommended to expand the access to high-technology specialist stroke care (Class II, Level B).

\section{References}

1. Langhorne P, Dennis M. Stroke Units: An Evidence based Approach. London: BMI Books, 1998

2. Stroke Unit Trialists' Collaboration. Organised in-patient (stroke unit) care for stroke. Cochrane Database of Systematic Reviews 2007, Issue 4. Art. no. CD000197. DOI: 10.1002/14651858.CD000197. pub2

. Stegmayr B. Stroke units in their natural habitat: Can results of randomised trials be reproduced in routine clinical practice? Stroke 1999;30:709-714.

4. Rudd G, Hoffman I, Irwin P, Lowe D, Pearson M. Stroke unit care and outcome: The 2001 National Sentinel Audit of Stroke. Stroke 2005; 36:103-106.

5. Seenan P, Long M, Langhorne P. Stroke units in their natural habitat: Systemic review of observational studies. Stroke 2007;38:1886-1892.

\section{Stroke services and pathways in South Africa \\ 5.1 Existing models of stroke care}

South African facilities and health resources and patient access to these resources vary widely within the health care system, depending on location and historical factors. In South Africa, the stroke unit model of care has not been widely implemented despite robust evidence of efficacy. Stroke is usually managed as part of general medical service where there are no dedicated beds or service assigned to stroke. Frequently, there are no minimum requirements for treatment stipulated, and protocols for stroke care have not been developed at most hospitals as stroke is not seen as a strategic priority owing to lack of human resources and funding. Shortages and pressure for hospital beds frequently result in stroke patients being discharged too early. For the post-acute phase of stroke care, neuro-rehabilitation centres are in short supply; moreover, they usually manage patients with traumatic brain injuries and spinal cord injuries as well as stroke, and bed demand invariably exceeds supply. Infrastructure for home-based care is fragmented and varies from province to province.

\subsection{Overcoming the gaps}

For improved stroke care, provincial health authorities will need to re-organise existing resources within district and secondary hospitals in accordance with national guidelines and the National Department 
of Health stroke initiative in order to provide a service with defined protocols for stroke care. Established units such as those in the public sector of the Western Cape could serve as a model for the re-organisation of existing resources to provide more effective stroke care without necessarily incurring additional cost. The stroke unit model at G F Jooste Hospital has demonstrated efficacy in reducing mortality after implementation. ${ }^{1}$ Local protocols should be developed according to available resources to guide the delivery of stroke services at a particular health care facility.

Stroke services should be organised so as to recognise the special medical, social and rehabilitative needs of stroke patients in specific sub-groups (e.g. paediatric and young adult patients) and be tailored to accommodate the cultural and linguistic diversity of the population. An effective stroke service requires the establishment of a seamless network consisting of acute stroke units, post-acute care and rehabilitation, as well as further care in the community. Problems of limited infrastructure and shortage of staff need to be addressed at national, provincial and district levels.

\subsection{Proposals for stroke care within the SA health care system}

The public health care system is stratified into different levels of care, and the following proposals for stroke care are based on the current guidelines for stroke care applied to the categories of health facilities defined by the National Department of Health (October 2006.)

\subsubsection{Facilities providing predominantly ambulatory care}

The aspects of stroke care listed below should be available at predominantly ambulatory care facilities. Medical and nursing staff, health promoters and community health workers at these facilities should have specific training for:

- awareness of risk factors for stroke

- recognition of symptoms and signs of stroke and transient ischaemic attack (TIA)

- implementation of primary preventive measures to reduce stroke incidence

- implementation of secondary preventative measures to reduce incidence of stroke after TIA or stroke

- established protocols of referral for selected patients to higher level of care according to defined criteria.

\subsubsection{Facilities providing inpatient services Level 3 hospital}

A facility that provides specialist and sub-specialist care as defined for level 3 services. Recommendations for a level 3 facility are a comprehensive stroke unit and service requirements as follows:

- all essential components of a stroke unit (as defined in paragraph 4.2)

- staffing to include stroke specialists (specialist physicians or neurologists trained in stroke care, neurosurgical service) and full multidisciplinary team (all disciplines)

- 24-hour comprehensive laboratory service including haematology with clotting profile

- full neuroradiology service (CT, MRI with software for diffusionweighted and MRA images, angiography, duplex Doppler carotid sonography)

- catheter laboratory facility with stroke interventionist available for endovascular procedures

- focused vascular surgery available (carotid, coronary, peripheral)

- full cardiac service including transthoracic echocardiogram and trans-oesophageal echocardiography
- protocols for:

- intravenous and intra-arterial interventional (thrombolysis) management of acute ischaemic stroke with supportive management in stroke unit as per protocol with high-care monitoring available for first 24 - 48 hours

- investigation and management of stroke in the young patient

- investigation and management (including neurosurgical) of intracranial haemorrhage.

- In a resource-constrained health care environment, examples of patients likely to be referred to a level 3 comprehensive stroke unit would be younger stroke patients with unknown cause for stroke; patients with symptom onset to predicted time of arrival at a level 3 hospital to be less than 3.5 hours (i.e. patient who could benefit from thrombolysis - neurosurgical and haematological backup required); patients with suspected cardio-embolic stroke (e.g. cardiac causes not due to atrial fibrillation and not previously investigated for cardiac disease); patients with TIAs (especially crescendo TIAs) presenting within 48 hours (urgent investigation to prevent stroke); and patients who require urgent CT scan if unavailable at the level 2 or level 1 hospital.

Ideally, every patient with stroke should have a CT scan as part of the management, but in reality this not always feasible because of logistic and resource constraints in many parts of the country. However, under certain circumstances, patients may well require referral to another centre for an urgent CT or MRI brain scan.

Indications for URGENT CT scan include: (i) depressed level of consciousness for which the cause is uncertain; (ii) suspected subarachnoid haemorrhage or cerebellar haematoma; (iii) if the diagnosis is in doubt - to exclude treatable causes e.g. subdural haematoma, space-occupying lesion or other mimics of stroke; (iv) if anticoagulants (e.g. patient in atrial fibrillation) or thrombolytic therapy are planned - for immediate detection of intracerebral haematoma or haemorrhagic infarct; $(v)$ worsening neurological deficits; $(v i)$ history or clinical findings suggestive of trauma; and (vii) ongoing seizures.

Level 2 hospital (regional)

This is a facility that provides care requiring the intervention of specialists as well as general practitioner services. Recommendations for a level 2 stroke unit are:

- all essential components of a stroke unit (as defined in paragraph 4.3)

- internal medicine specialist cover trained in stroke care

- CT scan facility on site with radiology cover to interpret scans

- essential investigations available: electrocardiogram (ECG), chest $\mathrm{X}$-ray, basic laboratory service for FBC, erythrocyte sedimentation rate (ESR), international normalised ratio (INR), syphilis testing (RPR and VDRL), blood sugar, urea and electrolytes. Level 2 hospitals should have easy access to echocardiography.

- Stroke patients who require investigation and management by a team led by a specialist physician will probably be managed at a level 2 unit. Examples of patients who should ideally be managed in a level 2 unit include: patients with cardio-embolic stroke where the cardiac cause is known and the local physician assesses the need and timing of anticoagulation; stroke patients with no cause or obvious risk factors for stroke that can be investigated on site; and stroke patients with depressed level of consciousness for which the cause is confirmed on CT scan and who do not require neurosurgical or other level 3 intervention.

- Certain level 2 regional hospitals (e.g. those with 24-hour CT scan on site and physicians able to interpret acute stroke scan, 24-hour laboratory service, and neurosurgical cover) could develop protocols for intravenous thrombolysis for acute ischaemic stroke. 
- Level 2 stroke units should have a protocol for transfer of selected stroke patients to a level 3 facility. Reasons for referral may include:

- selected patients who may benefit from thrombolysis and can reach a level 3 hospital within 3 hours of onset of symptoms of stroke.

- where urgent CT scan is required and is not currently available at the level 2 hospital (see indications above). Should CT on such patients reveal a subarachnoid haemorrhage or any lesion (including a lobar haemorrhage or cerebellar infarct or haemorrhage) with significant mass effect, then referral of the patients to neurosurgery is indicated before they can return to the level 2 hospital.

- patients with CT scans done at level 2 who show a subarachnoid haemorrhage or any other lesion (including a lobar haemorrhage or cerebellar infarct or haemorrhage) with significant mass effect on scan that requires neurosurgical intervention. Decompression may also be considered for the malignant middle cerebral artery syndrome.

- stroke in young patients for which no cause can be found.

- suspected cardio-embolic stroke (not associated with atrial fibrillation and not previously investigated for cardiac disease)

- patients with TIA onset within 48 hours of presentation require urgent vascular assessment (and carotid Doppler or vascular imaging not available at level 2)

- when a physician at level 2 facility requests further investigations not available at level 2 to determine cause of stroke (e.g. stroke due to a suspected arterial dissection)where there are large distances between level 3 and level 2 hospitals, the use of telemedicine or other telecommunication links should be explored

- all level 2 hospitals that do not meet the requirements listed above should have a level 1 stroke unit or service.

Level 1 hospital

A facility at which a range of outpatient and inpatient facilities are offered, where patients have conditions that can be managed by a medical officer or a team led by a family physician. Level 1 hospitals typically do not provide on-site cranial CT scanning. The recommendations for level 1 stroke unit or service are:

- minimumstaffing requirements:medical,nursing and physiotherapy personnel trained in stroke care

- comprehensive assessment of medical problems, impairments and disabilities

- established pathways and protocols for acute and post-acute management of stroke with careful attention to active management of physiological abnormalities to maintain homeostasis

- early initiation of rehabilitation plan involving the carers

- scheduled patient and family education about management, rehabilitation programme, causes of stroke, secondary prevention and available community resources

- early assessment and planning of discharge needs

- initiation of secondary prevention strategies

- ongoing staff training and education in stroke care

- protocol for referral and transfer of selected stroke patients to a level 2 or 3 facility: Where there are large distances between level 1 and level 2 or level 3 hospitals, the use of telemedicine or other telecommunication links should be explored.

Reasons for referral may include: (i) selected patients who may benefit from thrombolysis and can reach a level 3 hospital within 3 hours - refer direct to level 3; (ii) where urgent $\mathrm{CT}$ scan is required (see above); (iii) patients with suspected cardio-embolic stroke; (iv) stroke patients with depressed level of consciousness; $(v)$ all stroke patients with suspected intra-cranial haemorrhage; $(v i)$ younger stroke patients with unknown cause of stroke in whom family physician at level 1 requests further investigations not available at level 1 to determine cause of stroke; (vii) patients with suspected posterior fossa haemorrhage or infarct who may require surgical decompression; and (viii) patients with TIA onset within 48 hours of presentation.

\section{Reference}

1. de Villiers L, Kalula SZ, Burch VC. Does multidisciplinary stroke care improve outcome in a secondarylevel hospital in South Africa. Int J Stroke 2009;4(2):89-93.

\section{Primary stroke prevention}

Primary preventive measures reduce stroke incidence and should be universally available and actively promoted at all levels of health care in South Africa. ${ }^{1}$

\subsection{Lifestyle characteristics identified as a risk factors for stroke \\ 6.1.1 Smoking}

- Cigarette smoke is an independent risk factor for ischaemic stroke in men and women. ${ }^{13}$

- Smoking approximately doubles the risk of ischaemic stroke compared with non-smokers. ${ }^{13}$

- Smoking cessation reduces risk by $50 \%$ over 1 year and continues to decline, returning to baseline after 5 years. ${ }^{14}$

\subsubsection{Body weight and body fat distribution}

- Increased body weight status defined by a BMI $\left(\mathrm{kg} / \mathrm{m}^{2}\right)>25$ and increased abdominal fat (central obesity as measured by increased waist circumference $>94 \mathrm{~cm}$ in men and $>80 \mathrm{~cm}$ in women) has been associated with stroke risk in white people of European origin regardless of where they live in the world. These measurements vary for ethnic groups with lower waist circumference measurements applicable to South Asians, Japanese and people of Chinese decent. Specific data for Sub-Saharan Africans are currently not available. ${ }^{2-5}$

- Weight reduction is recommended and may lower blood pressure, thereby reducing risk of stroke. ${ }^{6}$

\subsubsection{Physical activity}

Exercise has beneficial effects on several important stroke risk factors and is associated with a reduction in stroke risk. ${ }^{?}$

\subsubsection{Nutrition}

- Diets rich in vegetables and fruits and with reduced sodium and increased potassium reduce stroke risk. ${ }^{8,9}$

- Specific diets lower blood pressure; these include the DASH diet (emphasis on vegetables and fruit, low-fat dairy products and a reduction in saturated and total fat) and diets low in sodium and rich in potassium. ${ }^{10-12}$

\subsubsection{Alcohol}

Light-to-moderate consumption ( $\leq 2$ drinks per day for men and $\leq 1$ drink per day for women) has been associated with reduced stroke risk. Heavier alcohol consumption ( $>60 \mathrm{ml} /$ day) increases the risk of both ischaemic (RR 1.69) and haemorrhagic stroke (RR 2.18). ${ }^{15}$

\section{Recommendations}

- Primary preventive measures reduce stroke incidence (Class III, Level A) 
- Abstention from smoking or smoking cessation (Class III, Level B).

- Weight reduction is recommended for those individuals with a BMI $>25 \mathrm{~kg} / \mathrm{m}^{2}$ (Class III, Level B).

- Regular physical activity $\geq 30$ minutes of moderate-intensity exercise daily (Class III, Level B).

- A diet low in sodium and rich in vegetables, fruits, lower total fat and saturated fat intake (Class III, Level B).

- Men should consume $\leq 2$ and non-pregnant women $\leq 1$ units (12 $\mathrm{ml}$ ) alcohol/day, and heavy use of alcohol is discouraged (Class III, Level B).

\subsection{Medical conditions identified as risk factors for stroke \\ 6.2.1 Hypertension}

- High blood pressure ( $\geq 130 / 85 \mathrm{mmHg}$ ) is the most important and prevalent modifiable risk factor for stroke.

- Significant reduction of stroke incidence occurs with a decrease in blood pressure. ${ }^{16,17}$

\subsubsection{Diabetes mellitus}

- Diabetes is an independent risk factor for ischaemic stroke.

- In patients with diabetes mellitus, tight blood pressure control and therapy with a statin reduces the risk of stroke. ${ }^{18,19}$

- Treatment of hypertension should where possible include an angiotensin-converting enzyme inhibitor or angiotensin receptor antagonist (usually in combination with a diuretic). ${ }^{17}$

- Clear evidence showing that risk of stroke is reduced by tight glycaemic control is lacking.

\subsubsection{Dyslipidaemia}

- The assessment of dyslipidaemia is best done in a fasting state to evaluate triglyceride, total cholesterol, HDL cholesterol and LDL cholesterol.

- Although the role of dyslipidaemia in ischaemic stroke causation, unlike its role in coronary artery disease, is not clear from epidemiological studies, it is associated with atherosclerosis - an important cause of stroke. Moreover, following an ischaemic stroke, lipid-lowering therapy is clearly beneficial.

- A thorough clinical assessment includes lifestyle and family history as well as a search for secondary causes that should be identified and addressed (for hypercholesterolaemia, conditions such as hypothyroidism, nephrotic syndrome and a fat-rich diet need to be considered; for hypertriglyceridaemia, consider diabetes, alcohol abuse, renal failure and hypothyroidism. Some medications may also affect the lipid profile adversely, though few do so profoundly.

- Diet influences plasma concentrations of cholesterol through the intake of cholesterol and saturated fat. Triglyceride intake in general should be limited in hypertriglyceridaemias. Statins are the drugs of choice in hypercholesterolaemias, but some mixed hyperlipidaemias and almost all hypertriglyceridaemias respond well to diet and fibrates.

- In high-risk patients with vascular disease and total cholesterol $>3.5 \mathrm{mmol} / \mathrm{l}$, taking a statin is associated with reduced ischaemic stroke (and myocardial infarction) rates. ${ }^{19}$

- For primary prevention, drug treatment is advised for persons with monogenic disorders and those with a high global risk score. ${ }^{20}$ This measure takes into account age, gender, total cholesterol (or LDLC), HDL cholesterol, smoking and blood pressure. Owing to the high risk for cardiovascular disease in diabetes mellitus, this has become a secondary prevention equivalent. Manifest atherosclerosis justifies treatment of the dyslipidaemia and is considered secondary prevention.

- Severe dyslipidaemia should be evaluated by a specialist physician to diagnose the monogenic disorders and consider less common secondary causes (referral criteria include: total cholesterol $>7.5$, LDLC $>5$, HDLC $>2.5$ or triglyceride $>5 \mathrm{mmol} / \mathrm{l}$. Urgent attention and referral is necessary for hypertriglyceridaemia $(>15 \mathrm{mmol} / \mathrm{l})$, low cholesterol $(<1.5 \mathrm{mmol} / \mathrm{l})$ or low HDL cholesterol $(<0.8$ $\mathrm{mmol} / \mathrm{l})$. The presence of tendon or cutaneous xanthomas should also be considered as reasons for referral, or significant adverse effects with lipid-modifying medication.

\subsubsection{Cardiac disease}

- Patients with mechanical heart valves, and atrial fibrillation with valvular heart disease, are at risk of future embolic events and should be anticoagulated, provided there are no clinically significant contraindications to anticoagulants. ${ }^{21,22}$

- Antiplatelet therapies reduce the risk of stroke in patients with atrial fibrillation but are less effective than anticoagulation. ${ }^{23}$

- The $\mathrm{CHADS}_{2}$ scoring system identifies patients with atrial fibrillation for primary stroke prevention reliably and allows selection of appropriate anticoagulant therapy (Table I). ${ }^{24,25}$

\section{Recommendations}

- Hypertension should be managed with lifestyle modification and pharmacotherapy (choice of regimen individualised and should follow SA Hypertension Society guidelines ${ }^{17}$ ) (Class I, Level A).

- Blood glucose should be checked regularly. Diabetes should be managed with lifestyle modification and individualised pharmacological therapy (Class IV, Level C). In diabetic patients, high blood pressure should be managed intensively (Class I, Level A) aiming for levels $<130 / 80 \mathrm{mmHg}$ (Class IV, Level C). Where possible, treatment should include an angiotensin-converting enzyme inhibitor or angiotensin receptor antagonist (Class I, Level A).

- For primary prevention, patients with monogenic disorders (evidenced by severe dyslipidaemia) or with type 2 diabetes or type 1 diabetes with micro-albuminuria, or those with a high global risk score, require dietary measures and lipid modifying treatment (Class I, Level A).

- Considering patient preferences, bleeding risk and access to reliable INR monitoring, the following patients should be considered for anticoagulation therapy with warfarin: patients with mechanical heart valves (target INR 2.5 - 3.5), patients with valvular heart disease and atrial fibrillation, and patients with a $\mathrm{CHADS}_{2}$ score indicating moderate or greater risk of stroke (target INR 2.0 - 3.0) (Class I, Level A). Lower-risk patients with atrial fibrillation or those in whom oral anticoagulation is clinically contraindicated should be considered for aspirin therapy (75 - $300 \mathrm{mg} /$ day).

\subsection{Other primary stroke prevention interventions}

\subsubsection{Aspirin for primary stroke prevention}

Low-risk subjects

- Six large randomised trials have evaluated the benefits of aspirin for the primary prevention of CV events in men and women (47 293 on aspirin, 45580 controls) with a mean age of 64.4 years. $^{26-31}$ Aspirin reduced coronary events and CV events, but not stroke, $\mathrm{CV}$ mortality and all-cause mortality. ${ }^{32}$ 
Table I. Non-valvular atrial fibrillation risk stratification and treatment recommendations: Risk stratification by (modified) $\mathrm{CHADS}_{2}$ scheme

\begin{tabular}{llll}
\hline $\begin{array}{l}\text { CHADS }_{2} \\
\text { score }\end{array}$ & Risk level & Stroke rate & $\begin{array}{l}\text { Treatment } \\
\text { recommendations }\end{array}$ \\
\hline & Low & $1.0 \% / \mathrm{yr}$ & $\begin{array}{l}\text { Aspirin }(75-325 \\
\mathrm{mg} / \mathrm{d})\end{array}$ \\
0 & $\begin{array}{l}\text { Low- } \\
\text { moderate }\end{array}$ & $1.5 \% / \mathrm{yr}$ & $\begin{array}{l}\text { Warfarin INR 2 - 3 } \\
\text { or aspirin (75 mg - } \\
325 \mathrm{mg} / \mathrm{d})\end{array}$ \\
& Moderate & $2.5 \% / \mathrm{yr}$ & Warfarin INR \\
2 & High & $5.0 \% / \mathrm{yr}$ & Warfarin INR 2 - 3 \\
3 & Very high & $>7 \% / \mathrm{yr}$ & Warfarin INR 2 - 3
\end{tabular}

Congestive heart failure, hypertension, age $>75$ yrs or diabetes $=1$ point each

${ }^{*}$ The $\mathrm{CHADS}_{2}$ scheme should be applied for primary prevention. ${ }^{\dagger}$ Consider patient preferences, bleeding risk and access to good INR monitoring. For those with a $\mathrm{CHADS}_{2}$ score $=1$, the number needed to treat to prevent 1 stroke over 1 yr with warfarin is $\approx 100$; excellent anticoagulation control is essential to achieve this benefit.

- No data are currently available on the use of other antiplatelet agents in primary prevention in low-risk subjects.

Subjects with vascular risk factors

- A systematic review of randomised studies comparing antithrombotic agents with placebo in patients with elevated BP and no prior $\mathrm{CV}$ disease showed that aspirin did not reduce stroke or total cardiovascular events. ${ }^{33}$

- Patients with atherosclerotic arterial disease have an increased risk of myocardial infarction, stroke and cardiovascular death. Aspirin reduces myocardial infarction in patients with asymptomatic carotid artery disease, and reduces stroke after carotid artery surgery. ${ }^{34,35}$

\subsubsection{Vitamins for primary stroke prevention}

- A low intake of vitamin D is associated with increased risk of stroke, but supplements of calcium plus vitamin D do not reduce the risk of stroke. ${ }^{36,37}$

- Supplements of tocopherol and beta carotene do not reduce stroke. ${ }^{38} \mathrm{~A}$ meta-analysis of trials with vitamin E supplementation found that it might increase mortality when used at high doses $(>400 \mathrm{IU} / \mathrm{d}){ }^{39}$

- High homocysteine levels are associated with increased stroke risk. However, a recent Cochrane systematic review did not find any evidence to suggest that dietary supplementation with folic acid or other B vitamins reduced stroke risk. ${ }^{40,41}$

- Folic acid and vitamin $B_{12}$ supplementation (with or without the addition of vitamin $\mathrm{B}_{6}$ ) does not reduce the risk of major $\mathrm{CV}$ events in patients with established vascular disease. ${ }^{42,43}$

\section{References}

1. Rothwell PM, Coull AJ, Howard SC, et al. Change in stroke incidence, mortality, case fatality, severity, and risk factors in Oxfordshire, UK from 1981 to 2004 (Oxford Vascular Study). Lancet 2004;363:19251933.
2. Alberti KG, Zimmet P, Shaw J. The International Diabetes Federation Epidemiology Task Force Consensus Group: The metabolic syndrome: a new worldwide definition. Lancet 2005:366:1059-1062.

3. Rexrode KM, Hennekens $\mathrm{CH}$, Willett WC, et al. A prospective study of body mass index, weight change, and risk of stroke in women. JAMA 1997;277:1539-1545.

Kurth T, Gaziano JM, Berger K, et al. Body mass index and the risk of stroke in men. Arch Intern Med 2002;162:2557-2562.

5. Isozumi K. Obesity as a risk factor for cerebrovascular disease. Keio I Med 2004:53:7-11.

6. Neter JE, Stam BE, Kok FJ, Grobbee DE, Gelejinse JM. Influence of weight reduction on blood pressure: a meta-analysis of randomized controlled trials. Hypertension 2003;42:878-884

7. Pate RR, Pratt M, Blair SN, et al. Physical activity and public health: a recommendation from the Centers for Disease Control and Prevention and the American College of Sports Medicine. JAMA 1995;273:402-407.

8. Bazzano LA, Serdula MK, Liu S. Dietary intake of fruits and vegetable and risk of cardiovascular disease. Curr Atheroscler Rep 2003;5:492-499.

9. Steffen LM, Jacobs DR Jr, Stevens J, Shahar E, Carithers T, Folsom AR. Associations of whole-grain, refined-grain, and fruit and vegetable consumption with risks of all-cause mortality and incident coronary artery disease and ischemic stroke: the Atherosclerosis Risk in Communities (ARIC) Study. Am J Clin Nutr 2003;78:383-390.

10. Whelton PK, He J, Cutler JA, et al. Effects of oral potassium on blood pressure. Meta-analysis of randomized controlled clinical trials. JAMA 1997;277:1624-1632

11. Appel LJ, Moore TJ, Obarzanek E, et al. A clinical trial of the effects of dietary patterns on blood pressure. DASH Collaborative Research Group. N Engl J Med 1997;336:1117-1124.

12. Sacks FM, Svetkey LP, Vollmer WM, et al. Effects on blood pressure of reduced dietary sodium and the Dietary Approaches to Stop Hypertension (DASH) diet. DASH-Sodium Collaborative Research Group. N Engl J Med 2001;344:3-10

13. Shinton R, Beevers G. Meta-analysis of relation between cigarette smoking and stroke. BMJ 1989;298:789-794.

14. Wolf PA, D'Agostino RB, Kannel WB, Bonita R, Belanger AJ. Cigarette smoking as a risk factor for stroke: the Framingham Study. JAMA 1988;259:1025-1029.

15. Reynolds K, Lewis B, Nolen JD, Kinney GL, Sathya B, He J. Alcohol consumption and risk of stroke: a meta-analysis [correction appears in JAMA 2003;289:2798]. JAMA 2003;289:579-588.

16. Neal B, MacMahon S, Chapman N. Blood Pressure Lowering Treatment Trialists' Collaboration. Effects of ACE inhibitors, calcium antagonists, and other blood-pressure-lowering drugs: results of prospectively designed overviews of randomised trials. Lancet 2000;356:1955-1964.

17. Seedat YK, Croasdale MA, Milne FJ, et al; Guideline Committee, Southern African Hypertension Society; Directorate: Chronic Diseases, Disabilities and Geriatrics, National Department of Health. South African hypertension guideline 2006. S Afr Med J 2006;96(4) (pt 2):337-362.

18. Effect of intensive blood-glucose control with metformin on complications in overweight patients with type 2 diabetes (UKPDS 34). UK Prospective Diabetes Study (UKPDS) Group [correction appears in Lancet 1998:352:1558]. Lancet 1998:352:854-865.

19. Heart Protection Study Collaborative Group. MRC/BHF Heart Protection Study of cholesterol lowering with simvastatin in 20536 high-risk individuals: a randomised placebo-controlled trial. Lancet 2002;360:7-22.

20. Sever PS, Dahlof B, Poulter NR, et al. for the ASCOT investigators. Prevention of coronary and stroke events with atorvastatin in hypertensive patients who have average or lower-than-average cholesterol concentrations, in the Anglo-Scandinavian Cardiac Outcomes Trial-Lipid Lowering Arm (ASCOTLLA): a multicentre randomised controlled trial. Lancet 2003;361:1149-1158.

21. Cannegieter SC, Rosendaal FR, Briet E. Thromboembolic and bleeding complications in patients with mechanical heart valve prostheses. Circulation 1994;89:635-641.

22. Bonow RO, Carabello B, de Leon AC Jr, et al. ACC/AHA guidelines for the management of patients with valvular heart disease: a report of the American College of Cardiology/American Heart Association Task Force on Practice Guidelines (Committee on Management of Patients With Valvular Heart Disease). J Am Coll Cardiol 1998;32(5):1486-1588.

23. Hart RG, Benavente O, McBride R, Pearce LA. Antithrombotic therapy to prevent stroke in patients with atrial fibrillation: a meta-analysis. Ann Intern Med 1999;131:492-501.

24. Gage BF, van Walraven $C$, Pearce $L$, et al. Selecting patients with atrial fibrillation for anticoagulation: stroke risk stratification in patients taking aspirin. Circulation 2004;110:2287-2292.

25. Gage BF, Waterman AD, Shannon W, Boechler M, Rich MW, Radford MJ. Validation of clinical classification schemes for predicting stroke: results from the National Registry of Atrial Fibrillation. JAMA 2001;285:2864-2870.

26. Peto R, Gray R, Collins R, et al. Randomised trial of prophylactic daily aspirin in British male doctors. Br Med J (Clin Res Ed) 1988:296:313-316.

27. Steering Committee of the Physicians' Health Study Research Group: Final report on the aspirin component of the ongoing Physicians' Health Study. N Engl J Med 1989;321:129-135

28. ETDRS Investigators: Aspirin effects on mortality and morbidity in patients with diabetes mellitus. Early Treatment Diabetic Retinopathy Study report 14. JAMA 1992;268:1292-1300.

29. Hansson L, Zanchetti A, Carruthers SG, et al. Effects of intensive bloodpressure lowering and low-dose aspirin in patients with hypertension: principal results of the Hypertension Optimal Treatment (HOT) randomised trial. HOT Study Group. Lancet 1998;351:1755-1762.

30. de Gaetano G. Low-dose aspirin and vitamin $\mathrm{E}$ in people at cardiovascular risk: a randomised trial in general practice. Collaborative Group of the Primary Prevention Project. Lancet 2001;357:89-95.

1. Iso $\mathrm{H}$, Hennekens $\mathrm{CH}$, Stampfer $\mathrm{MJ}$, et al. Prospective study of aspirin use and risk of stroke in women Stroke 1999;30:1764-1771.

32. Bartolucci AA, Howard G: Meta-analysis of data from the six primary prevention trials of cardiovascular events using aspirin. Am J Cardiol 2006;98:746-750.

33. Berger JS, Roncaglioni MC, Avanzini F, Pangrazzi I, Tognoni G, Brown DL. Aspirin for the primary prevention of cardiovascular events in women and men: a sex-specific meta-analysis of randomized controlled trials. JAMA 2006;295:306-313.

34. Hobson RW 2nd, Krupski WC, Weiss DG. Influence of aspirin in the management of asymptomatic carotid artery stenosis. VA Cooperative Study Group on Asymptomatic Carotid Stenosis. J Vasc Surg 1993;17:257-263; discussion 263-265.

35. Engelter S, Lyrer P. Antiplatelet therapy for preventing stroke and other vascular events after carotid endarterectomy. Cochrane Database Syst Rev 2003:CD001458.

36. Marniemi J, Alanen E, Impivaara $\mathrm{O}$, et al. Dietary and serum vitamins and minerals as predictors of myocardial infarction and stroke in elderly subjects. Nutr Metab Cardiovasc Dis 2005;15:188-197.

37. Hsia J, Heiss G, Ren H, et al. Calcium/vitamin D supplementation and cardiovascular events. Circulation 2007;115:846-854.

38. Tornwall ME, Virtamo J, Korhonen PA, Virtanen MJ, Albanes D, Huttunen JK. Postintervention effect of alpha tocopherol and beta carotene on different strokes: a 6-year follow-up of the Alpha Tocopherol, Beta Carotene Cancer Prevention Study. Stroke 2004;35:1908-1913. 
39. Miller ER 3rd, Pastor-Barriuso R, Dalal D, Riemersma RA, Appel LJ, Guallar E. Meta-analysis: highdosage vitamin E supplementation may increase allcause mortality. Ann Intern Med 2005;142:37-46.

40. The Homocysteine Studies Collaboration: Homocysteine and risk of ischemic heart disease and stroke: a meta-analysis. JAMA 2002;288:2015-2022.

41. Marti-Carvajal AJ, Sola I, Lathyris D, Salanti G. Homocysteine lowering interventions for preventing cardiovascular events. Cochrane Database of Systematic reviews 2009, Issue 4. Art. No.: CD 00612. DOI:10.10.1002/14651858. CD006612.pub2.

42. Loscalzo J. Homocysteine trials - clear outcomes for complex reasons. N Engl J Med 2006;354:1629 1632 .

43. The Heart Outcomes Prevention Evaluation (HOPE) 2 Investigators. Homocysteine lowering with folic acid and B vitamins in vascular disease. N Engl J Med 2006;354:1567-1577.

\section{Pre-hospital stroke management \\ 7.1 Public awareness and education}

- Successful care of the stroke victim begins with recognition by the public and health professionals that stroke is an emergency, like myocardial infarction.

- Avoiding delay should be the major aim of the prehospital phase of acute stroke care.

- Most stroke patients do not receive adequate therapy because they do not reach hospital soon enough. ${ }^{1}$

- Delays during acute stroke management have been identified at different levels: ${ }^{2}$

- at the population level, owing to failure to recognise the symptoms of stroke and contact emergency medical services (EMS)

- at the level of EMS and emergency physicians, owing to a failure to prioritise transport of stroke patients

- at the hospital level, owing to delays in neuro-imaging and inefficient in-hospital care, including a lack of protocol-driven acute stroke care.

- The interval from symptom onset to first call for medical help is the predominant part of prehospital delay. ${ }^{3-6}$ Major reasons for delayed contact include lack of awareness of stroke symptoms and recognition of their severity, but also denial of the disease and the hope that symptoms will resolve.

- The foregoing suggests that educating the population to recognise stroke symptoms, and changing people's attitudes to acute stroke, may reduce the delay from stroke onset to EMS involvement.

- Education should also be directed to paramedics and emergency department staff to improve the accuracy of stroke identification and speed up transfer to hospital. ${ }^{7}$ Education of paramedics increases stroke knowledge and clinical and communication skills, and decreases prehospital delays. ${ }^{8}$

\subsection{Organisation of ambulance service}

- Immediately stroke symptoms are suspected, patients or their proxies should call EMS.

- Emergency ambulance services should be organised to enable prompt response with the aim of transporting suspected stroke patients without delay to the emergency room of the nearest hospital (preferably with a stroke unit) that can provide organised acute stroke care. ${ }^{9,10}$

- Patients with onset of stroke symptoms within 3 hours should be given priority in evaluation and transportation to a level 2 or level 3 hospital where protocols for thrombolytic therapy are available.

- Doctors who receive a call or consultation from a patient with suspected stroke should recommend or arrange immediate transportation to the nearest hospital providing organised acute stroke care and ultra-early treatment.

- Patients with suspected subarachnoid haemorrhage should be referred urgently to a hospital with neurosurgical facilities.

- The emergency numbers used by the community should be userfriendly and easily accessible. Every ambulance service should be provided with information regarding hospitals that are well equipped to care for stroke victims in their vicinity.

\subsection{Pre-hospital diagnosis of stroke}

- Inaccurate initial diagnosis by professional groups represents a major problem. ${ }^{11,12}$

- EMS should have validated algorithm of questions to diagnose stroke during the phone interview.

- There should be in-house training of all paramedics, particularly pertaining to diagnosis and pre-hospital care of stroke patients.

- Paramedics should be able to recognise symptoms and signs of stroke and diagnose stroke using simple instruments such as the Face-Arm-Speech-Test. ${ }^{13}$

\subsection{Pre-hospital care}

Protocols should be used to guide pre-hospital stroke care. These concern maintaining physiological homeostasis and management of early complications or co-morbidities of stroke, such as impaired consciousness, seizures, vomiting or haemodynamic instability. General measures may include:

- Assess airway, breathing, circulation and disability (though assessment of disability should not delay transfer to hospital).

- Record history of event, including time of onset, signs and symptoms, and previous medical, drug and social history. This information should be obtained from patient and/or informant; the informant should be encouraged to accompany the patient. All medication should be brought with the patient.

- Alert the nearest stroke unit if the patient is potentially suitable for thrombolysis.

- Patients should receive nil by mouth.

- Maintain airway; patients may require oxygen to maintain saturation over $95 \%$.

- Blood glucose should be measured if possible, and hypoglycaemia should be treated if present with intravenous glucose solution.

- Maintain haemodynamic stability: actively manage hypotension by giving saline and/or raising the foot of the trolley.

\section{Recommendations}

- Educational programmes to increase public awareness of stroke (Class II, Level B).

- Educational programmes to increase stroke awareness among health care professionals (paramedics and emergency physicians) (Class II, Level B).

- Immediate EMS contact and dispatch to the nearest hospital (preferably with a stroke unit) that can provide organised acute stroke care for patients with suspected stroke (Class II, Level B).

- Priority transport with advance notification to the receiving hospital for patients with onset of stroke symptoms within 3 hours (Class III, Level B).

- Ambulance personnel should be trained to recognise stroke using simple instruments such as the FAST test (Class IV, GCP).

- During transport of stroke patients to hospital, paramedics should maintain physiological homeostasis and manage early complications or co-morbidities of stroke, according to protocol (Class IV, GCP).

\section{References}

1. Crocco TJ, Grotta JC, Jauch EC, et al. EMS management of acute stroke -prehospital triage. Prehospital Emergency Care 2007;II(3):313-317

2. Kwan J, Hand P, Sandercock P. A systematic review of barriers to delivery of thrombolysis for acute stroke. Age Ageing 2004;33:116-121. 
3. Keskin O, Kalemoglu M, Ulusoy RE. A clinic investigation into prehospital and emergency department delays in acute stroke care. Med Princ Pract 2005;14:408-412.

4. Chang K, Tseng M, Tan T. Prehospital delay after acute stroke in Kaohsiung, Taiwan. Stroke 2004;35:700704

5. Yu RF, San Jose MC, Manzanilla BM, Oris MY, Gan R. Sources and reasons for delays in the care of acute stroke patients. J Neurol Sci 2002;199:49-54.

6. Mosley I, Nicol M, Donnan G, Patrick I, Kerr F, Dewey H. The impact of ambulance practice on acute stroke care. Stroke 2007;38:2765-2770.

7. Kwan J, Hand P, Sandercock P. Improving the efficiency of delivery of thrombolysis for acute stroke: a systematic review. QJM 2004;97:273-279.

8. Behrens S, Daffertshofer M, Interthal C, Ellinger K, van Ackern K, Hennerici M. Improvement in stroke quality management by an educational programme. Cerebrovasc Dis 2002;13:262-266.

. Kothari R, Sauerbeck L, Jauch E, Broderick J, Brott T. Solving the issue of patient arrival time. Stroke 2007;38:2219-2220.

10. Chang KC, Tseng MC, Tan TY. Pre-hospital delay after acute stroke in Kaohsiung, Taiwan. Stroke 2004;35(3):700-704.

11. Becker K, Fruin M, Gooding T, Tirschwell D, Love P, Mankowski T. Community based education improves stroke knowledge. Cerebrovascular Dis 2001;II(I):34-43.

12. Yoon SS, Byles J. Perceptions of stroke in the general public and patients with stroke: qualitative study. BMJ 2002;324:1065-1068.

13. Nor AM, McAllister C, Louw SJ, et al. Agreement between ambulance paramedic- and physicianrecorded neurological signs with Face Arm Speech Test (FAST) in acute stroke patients. Stroke 2004;35:1355-1359.

\section{Management of acute ischaemic stroke}

\subsection{Urgent management}

Acute stroke/TIA should be treated as a medical emergency and evaluated with minimum delay, regardless of severity of deficits. In-hospital delays and time loss are a problem, especially for patients who are potential candidates for thrombolysis. Ideally, such patients should be evaluated and have a CT brain scan within 30 minutes of arrival in the emergency unit. Emergency units must create efficient pathways and processes to identify and evaluate suspected stroke patients, with rapid triage.

\subsection{Clinical assessment}

Initial evaluation of a suspected stroke patient entails checking vital signs and stabilisation of the patient, followed by assessment of neurological deficit and co-morbidities. Goals of this assessment include:

- determining whether patient has had a stroke

- identifying whether or not the patient is a suitable candidate for emergency interventional therapy with agents such as tPA

- excluding stroke mimics (i.e. other conditions with stroke-like symptoms)

- identifying other conditions that require immediate intervention (e.g. hypoglycaemia - urgent blood glucose assessment and treat if hypoglycaemic)

- determining potential causes of the stroke for early secondary prevention.

The cornerstone of the clinical assessment remains the history, general examination and neurological examination.

\section{FAST test}

F -Facial movements: Ask the patient to smile or show teeth. Look for new asymmetry.

A - Arm movements: Ask the patient to lift both arms together and hold. Does one arm drift or drop?

S - Speech: If the patient attempts conversation, look for speech disturbance.

T - Time: Act quickly and document time of onset of stroke symptoms.

\subsubsection{Medical history}

- Important information to obtain includes:

- time of onset of symptoms of stroke (defined as when patient was last awake and symptom-free or known to be asymptomatic)
- past medical history, particularly history of cardiac disease, peripheral vascular disease, diabetes and hypertension

- risk factors for atherosclerosis

- history of drug abuse, trauma, pregnancy, migraine, seizures, infection.

\subsubsection{Clinical examination}

\section{General examination}

- Assessment of vital signs, that should also include blood sugar, pulse oximetry, and body temperature.

- Examination of head and neck for signs of trauma, seizure activity (contusions and tongue lacerations), carotid bruits.

- Cardiac and vascular examination.

- Respiratory, abdominal and skin examination may reveal co-morbidities that may be associated with stroke risk or cause.

Neurological examination

- Should be brief but thorough.

- It is enhanced by use of a formal stroke score or scale, such as the NIH Stroke Scale (NIHSS). ${ }^{1}$ The NIHSS provides important information about the severity of stroke and prognostic information, and the score may influence decisions about acute treatment. This scale can be performed with a reasonable degree of accuracy by practitioners in a broad range of specialties. ${ }^{2}$

- The mRS (modified Rankin score) is a simple disability score often used to measure stroke outcome. ${ }^{3}$

\subsection{Diagnostic tests}

Several tests should be done routinely on patients with suspected ischaemic stroke to identify conditions that may cause or mimic stroke or that may influence therapeutic options.

\subsubsection{All patients}

- blood glucose

- full blood count

- urea, creatinine, electrolytes

- ESR or CRP

- ECG

- chest X-ray

- oxygen saturation.

\subsubsection{Investigations that may be required on selected} patients

- INR if patient suspected or known to be on warfarin

- fasting serum lipids

- serological test for syphilis (RPR or VDRL)

- lumbar puncture

- echocardiogram: transthoracic or transoesophageal

- 24-hour cardiac Holter

- full hypercoagulation screen

- anticardiolipin antibody (both IgG and IgM), thrombophilia screen

- collagen screen

- hepatic function tests

- toxicology screen

- MRI/MRA scan (diffusion and perfusion sequences), MR venogram

- CT angiogram

- cerebral angiography

- carotid duplex Doppler ultrasonography of neck. 


\subsubsection{Brain imaging}

- Brain imaging distinguishes ischaemic stroke from intracranial haemorrhage (haemorrhage v. infarct cannot be reliably predicted on clinical grounds, and management differs) and identifies other stroke mimics (e.g. neoplasm).

- Non-contrast CT scan of the brain distinguishes reliably between haemorrhagic and ischaemic stroke and is the most cost-effective strategy for imaging acute stroke patients. ${ }^{4}$ Non-haemorrhagic infarcts (ischaemic strokes) may not be apparent on CT scan within the first 6 hours of onset.

- Ideally, all stroke patients should have CT brain scan to accurately determine accurately the type of stroke and the anatomy of the stroke (an important clue to aetiology). In reality, this not always feasible because of logistical reasons and resource constraints in many parts of the country. However, under certain circumstances, patients may well be required to be referred to another centre for an urgent CT or MRI scan of the brain (see 5.3.2 for indications for urgent CT scan).

- Diagnostic brain imaging must be performed immediately on arrival at the hospital for stroke patients who are potential candidates for thrombolysis, to facilitate prompt treatment.

- The brain imaging study (CT or MRI) should be interpreted by a physician with expertise in reading CT or MRI studies of the brain. Some centres prefer to use MRI scan as first-line routine investigation for acute stroke.

- Diffusion-weighted MRI (DWI) is more sensitive than CT for detection of early ischaemic changes, and is particularly useful in the diagnosis of posterior circulation stroke and lacunar or small cortical infarctions. A DWI MRI can be negative in patients with definite stroke. ${ }^{5}$

- MRI can also detect small and old haemorrhages for a prolonged period with T2 gradient echo sequences. ${ }^{6}$

- MRI is particularly useful in acute stroke patients with unusual presentations and uncommon aetiologies, or in whom a stroke mimic is suspected but not clarified on CT.

- MRI is less suited for agitated patients or for those who may vomit and aspirate.

- Vascular imaging may identify the site and cause of arterial obstruction, and identifies patients at high risk of stroke recurrence.

- Vascular imaging may be necessary to identify patients with tight symptomatic arterial stenosis who could benefit from carotid endarterectomy or, in highly selected individuals, cerebral artery angioplasty.

- Non-invasive imaging with colour-coded duplex imaging of the extracranial arteries, CT angiography (CTA) or contrastenhanced MR angiography (CE-MRA) should be available at level 3 hospitals.

- Carotid Doppler studies are an important tool in evaluating patients with ischaemic stroke, and should be available at level 2 and level 3 hospitals.

- Non-invasive approaches to visualising the vasculature are associated with less risk than intra-arterial angiography, which has a $1-3 \%$ risk of causing stroke in patients with symptomatic carotid lesions.?

- Digital subtraction angiography (DSA) may be needed in some circumstances, e.g. when other tests have been inconclusive.

- Transcranial Doppler ultrasound (TCD) is not routine and is currently available at only a few centres in South Africa.

- TCD may be useful for the diagnosis of abnormalities in the large cerebral arteries at the base of the skull, and can be used to identify right-to-left cardiac shunts. ${ }^{8}$

\subsection{Treatment}

\subsubsection{General supportive treatment to maintain} homeostasis and treatment of complications

The term 'general treatment' refers to treatment strategies aimed at stabilising the critically ill patient to control systemic problems that may impair stroke recovery. The management of such problems is a central part of stroke treatment. ${ }^{9}$

General treatment includes respiratory and cardiac care; fluid and metabolic management; BP control; the prevention and treatment of conditions such as seizures, venous thrombo-embolism, dysphagia, aspiration pneumonia, other infections and pressure ulceration, and occasionally management of elevated intracranial pressure.

Best level of care is in a stroke unit where patients are admitted to acute care under a specialist team.

\section{Monitoring}

- There is little direct evidence from RCTs to indicate how intensively monitoring should be carried out, but in stroke unit trials it was common practice to have a minimum of 4-hourly observations for the first 72 hours after stroke. ${ }^{10}$

- Vital physiological functions such as blood pressure, pulse, oxygen saturation, blood glucose, temperature and level of consciousness are monitored. Neurological status can be monitored using validated neurological scales such as the NIH Stroke Scale.

- Clinical trials using continuous telemetry suggest there may be some benefit from more intensive continuous monitoring in terms of improved detection of complications and reduced length of stay, but clinical outcomes are inconclusive. ${ }^{11,12}$

- In practice, more intensive monitoring is often provided for subgroups of patients, such as those with reduced consciousness, progressing neurological deficits or a history of cardiorespiratory disease.

- Close monitoring is also required for the first 24 hours after thrombolysis.

- More invasive monitoring procedures, such as central venous catheters or intracranial pressure monitoring, are used only in highly selected patient groups.

Airway protection and pulmonary function

- Adequate tissue oxygenation is important in the setting of acute cerebral ischaemia; supply supplemental oxygen if hypoxic. Common causes of hypoxia are partial airway obstruction, hypoventilation, aspiration pneumonia and atelectasis.

- Patients with decreased level of consciousness or signs of brainstem dysfunction are at greatest risk of airway compromise because of impaired oropharyngeal mobility and loss of protective reflexes.

- There is a risk of airway obstruction in patients with vomiting or oropharyngeal muscular weakness (as in severe, bilateral or posterior circulation stroke).

\section{Fluid balance}

- Many stroke patients are dehydrated on admission to hospital; this is associated with poor outcome. ${ }^{13}$

- Although clinical trial evidence is limited, delivery of intravenous fluids is commonly considered to be part of general management of acute stroke, particularly in patients at risk of dehydration owing to reduced consciousness or impaired swallowing.

- Patients should remain nil per mouth, and fluids provided through intravenous line until their swallowing is formally assessed

- Maintain normal hydration and monitor daily urine output accordingly.

- Normal saline $(0.9 \%)$ is recommended for fluid replacement during the first 24 hours after stroke.

Blood pressure

- Blood pressure is elevated in many patients with acute stroke and 
often drops spontaneously during the first days after stroke, even without specific medical treatment.

- Blood flow in the critical ischaemic penumbra of the brain (brain tissue that is potentially salvageable following stroke) is passively dependent on the mean arterial pressure, and lowering the mean arterial pressure may damage this area.

- There are no adequately sized RCTs guiding BP management. In the absence of reliable evidence from clinical trials, many clinicians have developed protocols for the management of extremely high BP.

- It is common practice in many centres to begin cautious blood pressure reduction when levels exceed $220 \mathrm{mmHg}$ systolic and 120 $\mathrm{mmHg}$ diastolic.

- Patients with markedly elevated BP above these levels may have their BP lowered; a reasonable goal would be to lower the BP by $15 \%$ in the first 24 hours after stroke.

- Avoid drastic rapid reduction in BP.

- Upper level of systolic BP in patients undergoing thrombolytic therapy is $180 \mathrm{mmHg}$.

- Avoid and treat hypotension.

Glucose metabolism

- Hyperglycaemia after acute stroke is associated with larger infarct volumes and poor functional outcome. ${ }^{14-16}$

- There is limited evidence as to whether active reduction of glucose in acute ischaemic stroke improves patient outcomes.

- At present, the routine use of insulin infusion regimes in patients with moderate hyperglycaemia cannot be recommended.

- It is common practice in stroke units to reduce blood glucose levels $>180 \mathrm{mg} / \mathrm{dl}(10 \mathrm{mmol} / \mathrm{l}) .{ }^{10}$

- Use of intravenous saline and avoidance of glucose solutions in the first 24 hours after stroke is common practice, and appears to reduce blood glucose levels. ${ }^{17}$

- Hypoglycaemia $(<2.8 \mathrm{mmol} / \mathrm{l})$ may mimic an acute ischaemic infarction, and should be treated by intravenous dextrose bolus or infusion of $10-20 \%$ glucose. $^{18}$

- Marked elevations in blood glucose levels should be avoided.

- Treat hyperglycaemia with insulin when blood glucose $>10$ $\mathrm{mmol} / \mathrm{l}$.

Body temperature

- Fever is associated with poorer neurological outcome after stroke. $^{19-21}$

- Fever increases infarct size in experimental stroke.

- A raised body temperature should prompt a search for infection and treatment where appropriate.

- There are no adequately sized trials guiding temperature management after stroke.

- It is common practice to treat fever (and its cause) when temperature $\geq 37.5^{\circ} \mathrm{C}$.

Swallowing and nutrition

- Nil per mouth until assessment of ability to swallow (as a matter of routine) because of high risk of aspiration.

- Before testing the patient for swallowing competence, observe for:

- wet phonation

- abnormal voluntary cough

- abnormal phonation quality

- reduced level of consciousness

- reduced laryngeal elevation or swallow.

- If any of the above signs of possible aspiration are present or if level of consciousness is impaired, patient should remain on nil per mouth (fluids IVI or nasogastric tube) until swallowing formally assessed (often done by speech therapist).
- All patients have their swallowing assessed before receiving aspirin. Aspirin could be delayed for 24 hours, so it is not necessary to put a nasogastric tube into every patient.

- Videofluoroscopic study may be required on selected patients, depending on above.

- Swallowing difficulties usually improve within a few weeks after stroke but can persist, requiring long-term intervention or alternative feeding strategies.

- Nasogastric tube may be required for feeding and administration of medication.

- Nutritional intake (nasogastric feeding or oral) to be assessed and reviewed daily.

\section{Recommendations}

- Organisation of in-hospital pathways and systems for acute stroke patients is recommended (Class III, Level C).

- Ancillary tests, as outlined in 8.3.1, are recommended (Class IV, GCP).

- All stroke patients should be treated in a stroke unit (Class I, Level A).

- Health care systems should ensure that acute stroke patients have access to high-technology medical and surgical stroke care when required (Class III, Level B).

- In patients with suspected stroke or TIA, urgent cranial CT (Class I) or MRI is recommended (Class II, Level A).

- If MRI is used, the inclusion of diffusion-weighted imaging (DWI) and T2-weighted gradient echo sequences is recommended (Class II, Level A).

- In patients with TIA, minor stroke or early spontaneous recovery, immediate diagnostic work-up, including urgent vascular imaging (carotid ultrasound, CT angiography, or MR angiography) is recommended (Class I, Level A).

- In patients with acute stroke and TIA, early clinical evaluation, including physiological parameters and routine blood tests, is recommended (Class I, Level A).

- Additional diagnostic examinations are necessary in selected patients, depending on the type of stroke and suspected aetiology (Level IV, GCP).

- All acute stroke (and TIA) patients should have a 12-lead ECG. (Class I, Level A).

- Stroke and TIA patients seen after the acute phase should have 24-hour Holter ECG monitoring when arrhythmias are suspected and no other causes of stroke are found (Class I, Level A).

- Intermittent monitoring of neurological status, pulse, blood pressure, temperature and oxygen saturation is recommended for 72 hours in patients with significant persisting neurological deficits (Class IV, GCP).

- Oxygen should be administered if $\mathrm{sPO}_{2}$ falls below 95\% (Class IV, GCP).

- Acute stroke patients should be on nil per mouth until assessment of ability to swallow (Class IV, GCP).

- Regularmonitoring offluidbalance and electrolytesis recommended in patients with severe stroke or swallowing problems (Class IV, GCP).

- Normal saline $(0.9 \%)$ is recommended for fluid replacement during the first 24 hours after stroke (Class IV, GCP).

- Routine blood pressure lowering is not recommended following acute stroke (Class IV, GCP).

- Cautious blood pressure lowering is recommended in patients with any of the following: extremely high BP $(>220 / 120 \mathrm{mmHg})$ on repeated measurements, or severe cardiac failure, aortic dissection or hypertensive encephalopathy (Class IV, GCP). 
- Abrupt BP lowering should be avoided (Class II, Level C).

- Low BP secondary to hypovolaemia or associated with neurological deterioration in acute stroke should be treated with volume expanders (Class IV, GCP).

- Monitoring serum glucose levels is recommended (Class IV, GCP).

- Treatment of serum glucose levels $>10 \mathrm{mmol} / \mathrm{l}$ with insulin titration is recommended (Class IV, GCP).

- Severe hypoglycaemia $<2.8 \mathrm{mmol} / \mathrm{l}$ should be treated with intravenous dextrose or infusion of $10-20 \%$ glucose (Class IV, GCP).

- The presence of pyrexia (temperature $>37.5^{\circ} \mathrm{C}$ ) should prompt a search for concurrent infection (Class IV, GCP).

- Treatment of pyrexia $\left(>37.5^{\circ} \mathrm{C}\right)$ with paracetamol, fanning and tepid sponging is recommended (Class III, Level C).

- Antibiotic prophylaxis is not recommended in immunocompetent patients (Class II, Level B).

\subsubsection{Specific treatment}

8.4.2.1 Intravenous thrombolysis for acute ischaemic stroke

- Intravenous thrombolytic therapy with recombinant tissue plasminogen activator (tPA) is an accepted therapy for acute ischaemic stroke within 4.5 hours of onset.

- Intravenous tPA should be administered at a hospital with rapid triage of stroke patients and established protocols for use of tPA (where there is a strict adherence to inclusion and exclusion criteria) and where good post-treatment care is available.

- Intravenous tPA $(0.9 \mathrm{mg} / \mathrm{kg}$ body weight, maximum $90 \mathrm{mg}$, with $10 \%$ of the dose given as a bolus and the remainder given by a 60-minute infusion) given within 3 hours after ischaemic stroke onset, significantly improves outcome. ${ }^{22-24}$

- The ECASS III trial has shown that intravenous tPA administered between 3 and 4.5 hours after symptom onset significantly improves clinical outcomes in patients with acute ischaemic stroke, compared with placebo. ${ }^{25}$

- Treatment benefit is time-dependent and the number needed to treat (NNT) to get one more favourable outcome drops from 4 during the first 90 minutes through to 7 at 3 hours, and towards 14 between 3 and 4.5 hours. ${ }^{24,25}$

- Thrombolytic therapy should only be given if the diagnosis is established by a physician with expertise in the diagnosis of stroke and who is aware of the risks of this treatment.

- Imaging of the brain (CT scan or MRI) must be done prior to treatment with tPA and assessed by physicians with expertise in reading and interpreting the imaging study and when haemorrhage is excluded.

- Imaging of the brain must be available 24 hours per day and available as an emergency on request.

- BP must be below $185 / 110 \mathrm{mmHg}$ before and for the first 24 hours after thrombolysis. Management of high BP is required as protocol deviations are associated with higher mortality rates. ${ }^{22,26,27}$

- A laboratory service should also be available 24 hours/day at the hospital.

- Inclusion criteria for treatment of acute ischaemic stroke with intravenous tPA:

- diagnosis of ischaemic stroke causing measurable neurological deficit

- neurological signs should not be clearing spontaneously

- neurological signs should not be minor and isolated

- stroke symptoms should not be suggestive of subarachnoid haemorrhage
- onset of symptoms should be $<4.5$ hours before beginning treatment, and caution should be exercised in treating a patient with major deficits (NIHSS score $\geq 20$ ).

National Institute of Neurological Disorders and Stroke (NINDS) exclusion criteria for intravenous tPA

- time of onset of symptoms unknown or $>3$ hours (data from ECASS III trial support 4.5 hour cut-off)

- minor or rapidly improving stroke symptoms

- CT or MRI signs of haemorrhage

- history of previous intracranial haemorrhage

- head trauma or prior stroke in previous 3 months

- myocardial infarction in the previous 3 months

- gastro-intestinal or urinary tract haemorrhage in previous 21 days

- major surgery in previous 14 days

- arterial puncture at a non-compressible site in the previous 7 days

- history of previous intracranial haemorrhage

- systolic BP >185 mmHg, diastolic $>110 \mathrm{mmHg}$. If $\mathrm{BP}$ can be lowered safely with antihypertensive agents, the patient may be eligible for treatment. Doctors must assess BP stability before starting rt-PA. As time is limited, some patients with markedly elevated BP cannot be managed adequately and still meet the 3-hour requirement.

- evidence of active bleeding or acute trauma on examination

- patient taking an oral anticoagulant or, if anticoagulant being taken, INR $>1.5$

- if patient received heparin in the previous 48 hours, a PTT must not be above the normal range

- platelet count $<100000 \times 10^{9} / 1$

- blood glucose $<2.7 \mathrm{mmol} / \mathrm{l}$

- seizure at stroke onset with post-ictal residual neurological impairment with uncertainty if deficit owing to ischaemic stroke

- CT scan shows multilobar infarction (hypodensity greater than a third of the cerebral hemisphere)

- patient or family members do not understand the potential risks and benefits of treatment.

\section{Other thrombolytic agents}

Clinical trials of streptokinase were halted prematurely because of unacceptably high rates of haemorrhage; this agent should not be used. Other intravenously administered thrombolytic agents have not been tested extensively for treatment of patients with acute ischaemic stroke and should be avoided in routine clinical practice outside the context of a clinical trial.

\section{Dosage of intravenous tPA}

The dose is $0.9 \mathrm{mg} / \mathrm{kg}$ (maximum $90 \mathrm{mg}$ ), with $10 \%$ given as an intravenous bolus and the rest infused intravenously over 1 hour. The use of intravenous streptokinase or other thrombolytic agents apart from $\mathrm{tPA}$ is not recommended.

8.4.2.2 Endovascular therapy

- There are no RCTs comparing intra-arterial tPA with intravenous tPA, which is the presently accepted standard of care.

- The limited trials (intra-arterial route) available are often characterised by inadequate controls, no good outcome studies, and small sample sizes. ${ }^{28}$

- The results of these studies are therefore not at present robust enough to warrant recommendation for use in routine care outside specialist units.

- The availability of intra-arterial thrombolysis should generally not preclude the intravenous administration of TPA in otherwise eligible patients. Intra-arterial thrombolysis with or without mechanical clot disruption is an option for treatment of selected 
patients who have a major ischaemic stroke of $\leq 6$ hours duration owing to occlusion of the middle cerebral or basilar artery who are not otherwise candidates for intravenous tPA. ${ }^{29-32}$ This type of treatment requires that the patient be at a specialist stroke centre with experienced stroke clinicians with immediate access to cerebral angiography and qualified interventionists.

- Patients who are evaluated within 6 hours of symptoms but who are ineligible to receive intravenous thrombolysis because of recent surgery or other procedures, may be candidates for intra-arterial thrombolysis. ${ }^{33,34}$

- Studies comparing standard intravenous tPA with a combined intravenous and intra-arterial approach have started. ${ }^{35}$

- It is uncertain as to whether or not the use of extraction devices (such as the Merci device) are better than intra-arterial delivery of TPA with catheter tip mechanical clot disruption.

- Recanalisation has been used as a surrogate for outcome, but outcome depends on when recanalisation occurs - late recanalisation may not be associated with good outcome but could lead to increased complications; more data are needed.

- Only a minority of stroke patients in South Africa are likely to qualify for intra-arterial thrombolysis at present (given the time limits, resource constraints, lack of comprehensive stroke units and availability of expertise) - and then at least 2 requirements will need to be met: arterial occlusion is proven and that the occlusion is reachable with interventional techniques.

8.4.2.3 Early antiplatelet treatment after acute ischaemic stroke

- Aspirin started within 48 hours of ischaemic stroke is safe and effective, resulting in a modest net benefit with significantly fewer recurrent strokes. Aspirin was tested in large RCTs in acute $(<48$ hours) stroke, and a significant reduction was seen in death and dependency and recurrence of stroke ( 9 fewer deaths or non-fatal strokes per 1000 in the first few weeks, and 13 fewer dead or dependent per 1000 after some weeks' or months' follow-up). ${ }^{36,37}$

- The use of other antiplatelet agents such as clopidogrel or dipyridamole in the setting of acute ischaemic stroke has not been evaluated.

- Aphase 3 trial for the glycoprotein-IIb-IIIa antagonist abciximab was stopped prematurely because of an increased rate of bleeding. ${ }^{39}$

8.4.2.4 Anticoagulation for acute ischaemic stroke

- Results of recent trials show that early administration of either heparin or low-molecular-weight heparin fail to show net benefit and are associated with an increased risk of bleeding complications. $^{36,39-41}$

- Early anticoagulation increased the risk of symptomatic haemorrhages, especially among persons with larger infarcts. ${ }^{42}$

- These medications are also associated with a risk of serious bleeding in other parts of the body, although the likelihood of bleeding appears to be lower than that associated with the administration of thrombolytic agents.

- Present data indicate that early administration of heparin or lowmolecular-weight heparin does not lower the risk of early recurrent stroke, including among patients with cardio-embolic stroke.

- Early administration of anticoagulants does not lessen the risk of early neurological worsening.

- Data are not sufficient to indicate whether anticoagulants might have efficacy among potentially high-risk groups such as persons with intracardiac or intra-arterial thrombi.

- The efficacy of urgent anticoagulation is not established for treatment of patients with vertebrobasilar disease or an arterial dissection.

- Most trials have not demonstrated the efficacy of anticoagulation in improving outcomes of acute ischaemic stroke.
- The role of anticoagulants as an adjunctive therapy in addition to pharmacological or mechanical thrombolysis has not been defined.

- Low-dose subcutaneous heparin or low-molecular-weight heparin should be considered for patients at high risk of deep-vein thrombosis or pulmonary embolism.

8.4.2.5 Neuroprotection for acute ischaemic stroke

Currently no clinical trials have shown benefit for treatment of acute ischaemic stroke with neuroprotective agents.

Intracerebral haemorrhage

This guideline does not include the management of intracerebral haemorrhage; this has been fully discussed in the American guideline. $^{43}$

\section{Recommendations}

- Intravenous tPA $(0.9 \mathrm{mg} / \mathrm{kg}$, maximum $90 \mathrm{mg})$ with $10 \%$ of dose given as a bolus followed by infusion lasting 60 minutes is recommended within 4.5 hours of onset of ischaemic stroke (Class I, Level A), provided there are no contraindications for the treatment, although treatment between 3 and 4.5 hours is currently not included in the South African labelling.

- In specialised (level 3) stroke centres, intra-arterial treatment of acute middle cerebral artery occlusion within $\leq 6$ hours of the event is an option (Class II, Level B).

- The availability of intra-arterial thrombolysis should generally not preclude the intravenous administration of tPA in otherwise eligible patients (Class I, Level A).

- Intra-arterial thrombolysis is recommended for acute basilar occlusion in selected patients (Class III, Level B), and intravenous thrombolysis for basilar occlusion is an acceptable alternative even after 3 hours (Class III, Level B).

- Aspirin (150 - $300 \mathrm{mg}$ loading dose) should be given within 48 hours after ischaemic stroke (Class I, Level A).

- If thrombolytic therapy is planned or given, aspirin or other antithrombotic therapy should not be initiated within 24 hours (Class IV, GCP).

- The use of other antiplatelet agents (single or combined) is not recommended in the setting of acute ischaemic stroke (Class III, Level C).

- The administration of glycoprotein-IIb-IIIa inhibitors is not recommended (Class I, Level A).

- The early administration of unfractionated heparin, low-molecularweight heparin or heparinoids is not recommended for the treatment of patients with acute ischaemic stroke (Class I, Level A).

- There is no recommendation to treat ischaemic stroke patients with neuroprotective substances (Class I, Level A).

\section{References}

1. Lyden P, Brott T, Tilley B, et al. Improved reliability of the NIH Stroke Scale using video training. NINDS TPA Stroke Study Group. Stroke 1994;25:2220-2226.

2. Goldstein LB, Samsa GP. Reliability of the National Institutes of Health Stroke Scale: extension to nonneurologists in the context of a clinical trial. Stroke 1997;28:307-310.

3. van Sweiten JC, Koudstaal PJ, Visser MC, Schouten HJ, van Gijn J. Interobserver agreement for the assessment of handicap in stroke patients. Stroke 1988;19:604-607.

4. Wardlaw JM, Keir SL, Seymour J, et al. What is the best imaging strategy for acute stroke? Health Technol Assess 2004;8:iii,ix-x,1-180.

Ay H, Oliveira-Filho J, Buonanno FS, et al. 'Footprints' of transient ischemic attacks: a diffusionweighted MRI study. Cerebrovasc Dis 2002;14:177-186

6. Dimigen $M$, Keir S, Dennis M, Wardlaw J. Long-term visibility of primary intracerebral hemorrhage on magnetic resonance imaging. J Stroke Cerebrovasc Dis 2004;13:104-108.

. Willinsky RA, Taylor SM, TerBrugge K, Farb RI, Tomlinson G, Montanera W. Neurologic complications of cerebral angiography: prospective analysis of 2,899 procedures and review of the literature. Radiology 2003;227:522-528.

8. Klötzsch C, Janssen G, Berlit P. Transesophageal echocardiography and contrast-TCD in the detection of a patent foramen ovale: experiences with 111 patients. Neurology 1994;44:1603-1606. 
9. Leys D, Ringelstein EB, Kaste M, Hacke W. The main components of stroke unit care: results of a European expert survey. Cerebrovasc Dis 2007;23:344-352.

10. Langhorne P, Pollock A. What are the components of effective stroke unit care? Age Ageing 2002;31:365371.

11. Sulter G, Elting JW, Langedijk M, Maurits NM, De Keyser J. Admitting acute ischemic stroke patients to a stroke care monitoring unit versus a conventional stroke unit: a randomized pilot study. Stroke 2003;34:101-104.

12. Cavallini A, Micieli G, Marcheselli S, Quaglini S. Role of monitoring in management of acute ischemic stroke patients. Stroke 2003;34:2599-2603.

13. Bhalla A, Sankaralingam S, Dundas R, Swaminathan R, Wolfe CD, Rudd AG. Influence of raised plasma osmolality on clinical outcome after acute stroke. Stroke 2000;31:2043-2048.

14. Baird TA, Parsons MW, Phanh T, et al. Persistent poststroke hyperglycemia is independently associated with infarct expansion and worse clinical outcome. Stroke 2003:34:2208-2214.

15. Baird TA, Parsons MW, Barber PA, et al. The influence of diabetes mellitus and hyperglycaemia on stroke incidence and outcome. J Clin Neurosci 2002;9:618-626.

16. Parsons MW, Barber PA, Desmond PM, et al. Acute hyperglycemia adversely affects stroke outcome: a magnetic resonance imaging and spectroscopy study. Ann Neurol 2002;52:20-28.

17. Gray CS, Hildreth AJ, Sandercock PA, et al. Glucose-potassium-insulin infusions in the management of post-stroke hyperglycaemia: the UK Glucose Insulin in Stroke Trial (GIST-UK). Lancet Neurol 2007;6:397-406.

18. Huff IS. Stroke mimics and chameleons. Emerg Med Clin North Am 2002;20:583-595

19. Reith J, Jorgensen HS, Pedersen PM, et al. Body temperature in acute stroke: relation to stroke severity, infarct size, mortality, and outcome. Lancet 1996;347:422-425

20. Castillo J, Davalos A, Noya M. Aggravation of acute ischemic stroke by hyperthermia is related to an excitotoxic mechanism. Cerebrovasc Dis 1999;9:22-27.

21. Hajat C, Hajat S, Sharma P. Effects of poststroke pyrexia on stroke outcome: a meta-analysis of studies in patients. Stroke 2000;31:410-414

22. The National Institute of Neurological Disorders and Stroke rt-PA Stroke Study Group: Tissue plasminogen activator for acute ischemic stroke. $\mathrm{N}$ Engl J Med 1995;333:1581-1587.

23. Wardlaw JM, Zoppo G, Yamaguchi T, Berge E. Thrombolysis for acute ischaemic stroke. Cochrane Database Syst Rev 2003:CD000213

24. Hacke W, Donnan G, Fieschi C, et al. Association of outcome with early stroke treatment: pooled analysis of ATLANTIS, ECASS, and NINDS rt-PA stroke trials. Lancet 2004;363:768-774.

25. Hacke W, Kaste M, Bluhmki E, et al. ECASS Thrombolysis with alteplase 3 to 4.5 hours after acute ischemic stroke. N Engl J Med 2008;359(13):1317-1329.

26. Katzan IL, Hammer MD, Furlan AJ, Hixson ED, Nadzam DM. Quality improvement and tissue-type plasminogen activator for acute ischemic stroke: a Cleveland update. Stroke 2003;34:799-800.

27. Graham GD: Tissue plasminogen activator for acute ischemic stroke in clinical practice: a meta-analysis of safety data. Stroke 2003;34:2847-2850

28. Furlan A, Higashida R, Wechsler L, et al. Intra-arterial prourokinase for acute ischemic stroke. The PROACT II study: a randomized controlled trial. Prolyse in Acute Cerebral Thromboembolism. JAMA 1999;282:2003-2011.

29. Higashida RT, Furlan AJ, Roberts H, et al. Technology Assessment Committee of the American Society of Interventional and Therapeutic Neuroradiology; Technology Assessment Committee of the Society of Interventional Radiology. Trial design and reporting standards for intra-arterial cerebral Society of Interventional Radiology. Trial design and reporting standards for intra-arterial cerebral
thrombolysis for acute ischemic stroke [published correction appears in Stroke 2003;34:2774]. Stroke thrombolysis for ac

30. Lee M, Hong K-S, Saver JL. Efficacy of intra-arterial fibrinolysis for acute ischemic stroke: Meta-analysis of randomized controlled trials. Stroke 2010;41;932-937.

1. Mattle HP, Arnold M, Georgiadis D, et al. Comparison of intraarterial and intravenous thrombolysis for ischemic stroke with hyperdense middle cerebral artery sign. Stroke 2008;39:379-383.

32. Nedeltchev K, Fischer U, Arnold M, et al. Long-term effect of intraarterial thrombolysis in stroke Stroke 2006;37:3002-3007.

33. Chalela JA, Katzan I, Liebeskind DS, et al. Safety of intra-arterial thrombolysis in the postoperative period. Stroke 2001;32:1365-1369.

34. Choi JH, Bateman BT, Mangla S, et al. Endovascular recanalization therapy in acute ischemic stroke. Stroke 2006;37:419-424.

35. IMS investigators: The Interventional Management of Stroke (IMS) II Study. Stroke 2007;38:21272135

36. International-Stroke-Trial-Collaborative-Group: The International Stroke Trial (IST): a randomised trial of aspirin, subcutaneous heparin, both, or neither among 19435 patients with acute ischaemic stroke. Lancet 1997;349:1569-1581.

37. CAST-Collaborative-Group: CAST: randomised placebo-controlled trial of early aspirin use in 20000 patients with acute ischaemic stroke. Lancet 1997;349:1641-1649.

\section{Management of neurological complications of acute ischaemic stroke}

\subsection{Acute brain oedema and raised} intracranial pressure

- Patients with large infarctions affecting the cerebral hemisphere or cerebellum are at high risk for complicating brain oedema and increased intracranial pressure.

- Close monitoring of a patient for signs of neurological worsening during the first few days after stroke is recommended.

- Basic management of stroke patients with raised intracranial pressure includes head positioning at an elevation of up to $30^{\circ}$, avoidance of noxious stimuli, pain relief, appropriate oxygenation and normalising body temperature.

- Malignant middle cerebral artery (MCA) syndrome. Patients with massive space-occupying hemispheric infarction have a poor prognosis. Life-threatening brain oedema usually develops between the 2 nd and 5 th day after stroke onset, but up to a third of patients can have neurological deterioration within 24 hours after symptom onset., ${ }^{1,2}$ Decompressive surgery for malignant oedema of the cerebral hemisphere (malignant middle cerebral artery syndrome) may be life saving. ${ }^{3,4}$ A pooled analysis of 93 patients from 3 trials that evaluated decompressive surgery for the treatment of malignant infarction of the middle cerebral artery showed that, compared with the control group that received conservative medical therapy, at 1 year the probability of survival increased from $28 \%$ in the control group to nearly $80 \%$ in patients in the decompressive surgery group, and the probability of survival with a mild or moderate disability (mRS $\leq 3)$ doubled ( $21 \%$ v. $43 \%)$. However, the probability of surviving in a condition requiring assistance from others ( $\mathrm{mRS}$ of 4 ) increases (2\% v. $31 \%$ ), although the risk of very severe disability (mRS of 5) is not increased (5\% v. $4 \%$ ) by decompressive surgery. ${ }^{3}$ Inclusion criteria for this combined analysis were age 18 - 60 years, NIHSSS $>15$, decrease in level of consciousness to a score $\geq 1$ on item 1a of the NIHSS, infarct signs on CT of $50 \%$ or more of the MCA territory or $>145 \mathrm{~cm}^{3}$ on DWI, and inclusion $<45$ hours after onset (surgery $<48$ hours). A systematic review of 12 observational retrospective studies found age $>50$ years to be a predictor of poor outcome. ${ }^{5}$ Even patients with aphasia may improve significantly. ${ }^{6}$ The decision to perform decompressive surgery in an individual patient with space-occupying hemispheric infarction will depend on the willingness to accept survival with moderate disability, the age of the patient, and subsequent access to rehabilitation. Many hospitals do not have neurosurgical expertise, and transfer of patients at risk for malignant brain oedema to an institution with such expertise should be considered, particularly for the younger patient.

- Medical management. There is no RCT evidence to support the use of medical therapy in patients with large space-occupying infarctions and brain oedema. However, some stroke clinicians consider the use of the following agents despite the lack of evidence: intravenous glycerol $(4 \times 250 \mathrm{ml}$ of $10 \%$ glycerol over 30 - 60 minutes) or mannitol (25 - 50 g every $3-6$ hours) ${ }^{7,8}$ Hypotonic and glucose-containing solutions should be avoided as replacement fluids. Dexamethasone and corticosteroids are not useful. ${ }^{9}$

- Large cerebellar infarctions. Large cerebellar infarctions may require urgent decompression (shunting or surgical evacuation). The operation should be performed before signs of herniation are present. The prognosis among survivors can be very good, even in patients who are comatose before surgery.

\subsection{Seizures}

- Seizures may occur in the acute phase of ischaemic stroke. Antiepileptic drugs should be used, based on general principles of seizure management.

- There is no evidence that primary prophylactic anticonvulsive treatment is beneficial.

\subsection{Agitation}

Agitation and confusion may be a consequence of acute stroke, but may also be due to complications such as fever, volume depletion or infection. Adequate treatment of the underlying cause must precede any type of sedation or antipsychotic treatment.

\section{Recommendations}

- Medical treatment including osmotherapy, for treatment of 
deteriorating patients with malignant brain oedema after large cerebral infarction is unproven (Class III, Level C).

- Hyperventilation is a short-lived intervention.

- Corticosteroids are not recommended for treatment of cerebral oedema because of lack of evidence of efficacy (Class III, Level A).

- It is recommended that ventriculostomy or surgical decompression be considered for treatment of large cerebellar infarctions that compress the brainstem (Class III, Level C).

- Decompressive surgery should be considered within 48 hours of symptom onset for patients with evolving malignant oedema of the cerebral hemisphere, but physicians should advise the patient's family about the potential outcomes including survival and disability (Class I, Level A). Both the age of the patient and the side of infarction may affect decisions about surgery. Age $>50$ years is a predictor of poor outcome.

- Prophylactic administration of anticonvulsants is not recommended.

- Recurrent seizures after stroke should be treated as in other neurological conditions.

References

Hacke W, Schwab S, Horn M, Spranger M, De Georgia M, von Kummer R. 'Malignant' middle cerebra artery territory infarction: clinical course and prognostic signs. Arch Neurol 1996;53:309-315.

2. Qureshi AI, Suarez II, Yahia AM, et al. Timing of neurologic deterioration in massive middle cerebral artery infarction: a multicenter review. Crit Care Med 2003;31:272-277.

3. Vahedi $\mathrm{K}$, Hofmeijer J, Jüttler $\mathrm{E}$, et al. Early decompressive surgery in malignant infarction of the middle cerebral artery: a pooled analysis of three randomised controlled trials. Lancet Neurol 2007;6:215-222. Jüttler E, Schwab S, Schmiedek P, et al. Decompressive surgery for the treatment of malignant infarction of the middle cerebral artery (DESTINY): a randomized, controlled trial. Stroke 2007;38:2518-2525.

Gupta R, Connolly ES, Mayer S, Elkind MS. Hemicraniectomy for massive middle cerebral artery territory infarction: a systematic review. Stroke 2004;35:539-543.

6. Kastrau F, Wolter M, Huber W, Block F. Recovery from aphasia after hemicraniectomy for infarction of the speech-dominant hemisphere. Stroke 2005;36:825-829.

7. Righetti E, Celani MG, Cantisani TA, Sterzi R, Boysen G, Ricci S. Glycerol for acute stroke: a Cochrane systematic review. J Neurol 2002;249:445-451.

8. Bereczki D, Liu M, do Prado GF, Fekete I. Mannitol for acute stroke. Cochrane Database Syst Rev 2001;1:CD001153.

9. Qizilbash N, Lewington SL, Lopez-Arrieta JM. Corticosteroids for acute ischaemic stroke. Cochrane Database Syst Rev 2002;2:CD000064.

\section{Management of common systemic complications of acute ischaemic stroke \\ 10.1 Prevention of DVT and PE}

- Early hydration and mobilisation can potentially reduce the risk of DVT and PE.

- Graded compression stockings have not been shown to be effective in preventing venous thrombo-embolism after stroke. Data from the CLOTS trial do not lend support to the use of thigh-length stockings in patients admitted to hospital with acute stroke. ${ }^{1}$

- Low-dose low-molecular-weight heparins reduced the incidence of both DVT and PE in stroke patients, without an increased risk of intracerebral or extracerebral haemorrhage (NNT: 7 and 38 for DVT and PE, respectively).

- Low-dose unfractionated heparin decreased the thrombosis risk but had no influence on pulmonary embolism. The risk of intracranial haemorrhage was not statistically significantly increased. ${ }^{2}$

- Prophylaxis with low-molecular-weight heparins or subcutaneous low-dose unfractionated heparin (5 $000 \mathrm{IU}$ twice daily) is indicated in patients at high risk of DVT or PE (e.g. due to immobilisation, obesity, diabetes, previous stroke). ${ }^{3,4}$

\subsection{Aspiration and pneumonia}

- Bacterial pneumonia is an important complication in stroke patients, and is mainly caused by aspiration. ${ }^{5,6}$
- Aspiration is frequently found in patients with reduced consciousness and in those with swallowing disturbances.

- Oral feeding should be withheld until the patient has demonstrated intact swallowing with small amounts of water and intact coughing on command. Nasogastric (NG) or percutaneous enteral gastrostomy (PEG) feeding may prevent aspiration pneumonia, although reflux of liquid feed, hypostasis, diminished cough and immobilisation increase the risk

- Frequent changes of the patient's position in bed and pulmonary physical therapy may prevent aspiration pneumonia.

\subsection{Dysphagia and feeding}

- Dysphagia occurs in up to $50 \%$ of patients with unilateral hemiplegic stroke.

- The prevalence of dysphagia is highest in the acute stages of stroke, and declines to around $15 \%$ at 3 months. ${ }^{8}$

- Dysphagia is associated with a higher incidence of medical complications and increased overall mortality.

- Withholding or limiting oral intake can worsen the catabolic state that may be associated with an acute illness such as stroke.

- Estimates of the incidence of malnutrition vary from 7 - 15\% at admission to $22-35 \%$ at 2 weeks. ${ }^{9-11}$

- Malnutrition predicts a poor functional outcome and increased mortality. ${ }^{12-14}$

- Routine supplementation for all acute stroke patients has not been shown to improve outcomes or reduce complications. ${ }^{15}$ There are no adequately powered trials of targeting supplementation to stroke patients at high risk of malnutrition.

- For patients with continuing dysphagia, options for enteral nutrition include NG or PEG feeding.

- A trial of early (median 48 hours post-stroke) versus delayed (1 week) NG feeding found no significant benefit of early feeding, although there was a trend to fewer deaths in the early NG group. ${ }^{15}$

- In a trial examining PEG and NG feeding within 30 days, PEG feeding was no better than NG and in fact was potentially harmful. ${ }^{15}$

- PEG feeding has also been studied in longer-term dysphagia, and two trials comparing PEG and NG feeding found a trend towards improved nutrition with PEG feeding that did not reach statistical significance. ${ }^{16,17}$

- Studies that have addressed quality of life found it was not improved by PEG feeding. ${ }^{18,19}$

\subsection{Pressure ulcers}

- In patients at high risk of developing pressure ulcers, use of support surfaces, frequent repositioning, optimising nutritional status, and moisturising sacral skin are appropriate preventive strategies. ${ }^{20}$ An air-filled or fluid-filled mattress may be useful for patients at high risk.

- The skin of the incontinent patient must be kept dry.

\subsection{Urinary tract infections and incontinence}

The majority of hospital-acquired urinary tract infections are associated with the use of indwelling catheters. ${ }^{21,22}$ Intermittent catheterisation has not been shown to reduce the risk of infection. Once urinary infection is diagnosed, appropriate antibiotics should be chosen. Prophylactic antibiotics are best avoided to prevent bacterial resistance developing.

- Urinary incontinence is common after stroke, particularly in older, more disabled and cognitively impaired patients, and is a strong predictor of poor functional outcome, even after correcting for age 
and functional status. ${ }^{23,24}$ However, data from the available trials are insufficient to guide continence care of adults after stroke. ${ }^{22,25}$

- Professional input through structured assessment and management of care and specialist continence nursing may reduce urinary incontinence and related symptoms after stroke and improve continence rates in both inpatients and outpatients. ${ }^{22,26}$

- Trials of interventions are insufficient in number and quality to make any firm recommendations. ${ }^{25}$

\subsection{Falls}

- Falls are common (up to 25\%) after stroke in the acute setting, during inpatient rehabilitation, and in the long term..$^{27-29}$

- Risk factors for falls in stroke survivors include cognitive impairment, depression, polypharmacy and sensory impairment. ${ }^{30-32}$

- A multidisciplinary prevention approach that focuses on personal and environmental factors has been found to be successful in general rehabilitation settings. ${ }^{33,34}$

\section{Recommendations}

- Infections after stroke should be treated with appropriate antibiotics (Class IV, GCP).

- Prophylactic administration of antibiotics is not recommended (Class II, Level B).

- Early rehydration is recommended to reduce the incidence of venous thromboembolism (Class IV, GCP).

- Graded stockings are not recommended.

- Early mobilisation is recommended to prevent complications such as aspiration pneumonia, DVT and pressure ulcers (Class IV, GCP).

- Low-molecular-weight heparins or low-dose subcutaneous heparin should be considered for patients at high risk of DVT or PE (Class I, Level A).

- In stroke patients with urinary incontinence, specialist assessment and management is recommended (Class III, Level C).

- Swallowing assessment is recommended, but there are insufficient data to recommend a specific approach for treatment (Class III, GCP).

- Early commencement of nasogastric (NG) feeding (within 48 hours) is recommended in stroke patients with impaired swallowing (Class II, Level B).

- Percutaneous enteral gastrostomy (PEG) feeding should not be considered in stroke patients in the first 2 weeks (Class II, Level B).

- Oral dietary supplements are only recommended for non-dysphagic stroke patients who are malnourished (Class II, Level B).

- An assessment of falls risk is recommended for every stroke patient (Class IV, GCP).

References

1. Effectiveness of thigh-length graduated compression stockings to reduce the risk of deep vein thrombosis after stroke (CLOTS trial 1): a multicentre, randomised controlled trial. The CLOTS Trials Collaboration. Lancet 2009;373:1958-1965.

2. Kamphuisen PW, Agnelli G, Sebastianelli M. Prevention of venous thromboembolism after acute ischemic stroke. J Thromb Haemost 2005;3:1187-1194.

3. Diener HC, Ringelstein EB, von Kummer R, et al. Prophylaxis of thrombotic and embolic events Diener $\mathrm{HC}$, Ringelstein $\mathrm{EB}$, von Kummer $\mathrm{R}$, et al. Prophylaxis of thrombotic and embolic events
in acute ischemic stroke with the low-molecular-weight heparin certoparin: results of the PROTECT Trial. Stroke 2006;37:139-144.

4. Sherman DG, Albers GW, Bladin C, et al. The efficacy and safety of enoxaparin versus unfractionated heparin for the prevention of venous thromboembolism after acute ischaemic stroke (PREVAIL Study): an open-label randomised comparison. Lancet 2007;369:1347-1355.

5. Weimar C, Roth MP, Zillessen G, et al. Complications following acute ischemic stroke. Eur Neurol 2002;48:133-140.

6. Horner J, Massey EW, Riski JE, Lathrop DL, Chase KN. Aspiration following stroke: clinical correlates and outcome. Neurology 1988;38:1359-1362.
7. Martino R, Foley N, Bhogal S, Diamant N, Speechley M, Teasell R. Dysphagia after stroke: incidence, diagnosis, and pulmonary complications. Stroke 2005;36:2756-2763.

8. Mann G, Hankey GJ, Cameron D. Swallowing function after stroke: prognosis and prognostic factors at 6 months. Stroke 1999;30:744-748.

9. Dennis MS, Lewis SC, Warlow C. Routine oral nutritional supplementation for stroke patients in hospital (FOOD): a multicentre randomised controlled trial. Lancet 2005;365:755-763.

10. Axelsson K, Asplund K, Norberg A, Alafuzoff I. Nutritional status in patients with acute stroke. Acta Med Scand 1988;224:217-224.

11. Axelsson K, Asplund K, Norberg A, Eriksson S. Eating problems and nutritional status during hospital stay of patients with severe stroke. J Am Diet Assoc 1989;89:1092-1096.

12. Finestone HM, Greene-Finestone LS, Wilson ES, Teasell RW. Prolonged length of stay and reduced functional improvement rate in malnourished stroke rehabilitation patients. Arch Phys Med Rehabil 1996;77:340-345.

13. Dávalos A, Ricart W, Gonzalez-Huix F, et al. Effect of malnutrition after acute stroke on clinical outcome. Stroke 1996;27:1028-1032

14. Food trial collaboration: Poor nutritional status on admission predicts poor outcomes after stroke: observational data from the FOOD trial. Stroke 2003;34:1450-1456.

15. Dennis MS, Lewis SC, Warlow C. Effect of timing and method of enteral tube feeding for dysphagic stroke patients (FOOD): a multicentre randomised controlled trial. Lancet 2005;365:764-772.

16. Norton B, Homer-Ward M, Donnelly MT, Long RG, Holmes GK. A randomised prospective comparison of percutaneous endoscopic gastrostomy and nasogastric tube feeding after acute dysphagic stroke. BMJ 1996;312:13-16

17. Hamidon BB, Abdullah SA, Zawawi MF, Sukumar N, Aminuddin A, Raymond AA. A prospective comparison of percutaneous endoscopic gastrostomy and nasogastric tube feeding in patients with acute dysphagic stroke. Med J Malaysia 2006;61:59-66.

18. Callahan CM, Haag KM, Weinberger M, et al. Outcomes of percutaneous endoscopic gastrostomy among older adults in a community setting. J Am Geriatr Soc 2000;48:1048-1054.

19. Rickman J. Percutaneous endoscopic gastrostomy: psychological effects. Br J Nurs 1998;7:723-729.

20. Reddy M, Gill SS, Rochon PA. Preventing pressure ulcers: a systematic review. JAMA 2006;296:974984.

1. Gerberding JL. Hospital-onset infections: a patient safety issue. Ann Intern Med 2002;137:665-670.

22. Thomas L, Cross S, Barrett J, et al. Treatment of urinary incontinence after stroke in adults. Cochrane Database Syst Rev 2008:CD004462.

23. Jorgensen L, Engstad T, Jacobsen BK. Self-reported urinary incontinence in noninstitutionalized long term stroke survivors: A population-based study. Arch Phys Med Rehabil 2005;86:416-420.

24. Meijer R, Ihnenfeldt DS, de Groot IJ, van Limbeek J, Vermeulen M, de Haan RJ. Prognostic factors for ambulation and activities of daily living in the subacute phase after stroke. A systematic review of the literature. Clin Rehabil 2003;17:119-129.

25. Dumoulin C, Korner-Bitensky N, Tannenbaum C. Urinary incontinence after stroke: does rehabilitation make a difference? A systematic review of the effectiveness of behavioral therapy. Top Stroke Rehabil 2005;12:66-76

26. Thomas LH, Barrett J, Cross S, et al. Prevention and treatment of urinary incontinence after stroke in adults. Cochrane Database Syst Rev 2005:CD004462.

27. Forster A, Young J. Incidence and consequences of falls due to stroke: a systematic inquiry. BM] 1995;311:83-86.

28. Mackintosh SF, Goldie P, Hill K. Falls incidence and factors associated with falling in older, communitydwelling, chronic stroke survivors (>1 year after stroke) and matched controls. Aging Clin Exp Res 2005;17:74-81.

29. Mackintosh SF, Hill KD, Dodd KJ, Goldie PA, Culham EG. Balance score and a history of falls in hospital predict recurrent falls in the 6 months following stroke rehabilitation. Arch Phys Med Rehabi 2006;87:1583-1589.

30. Lamb SE, Ferrucci L, Volapto S, Fried LP, Guralnik JM. Risk factors for falling in home-dwelling older women with stroke: the Women's Health and Aging Study. Stroke 2003;34:494-501.

31. Aizen E, Shugaev I, Lenger R. Risk factors and characteristics of falls during inpatient rehabilitation of elderly patients. Arch Gerontol Geriatr 2007;44:1-12.

32. Teasell R, McRae M, Foley N, Bhardwaj A. The incidence and consequences of falls in stroke patients during inpatient rehabilitation: factors associated with high risk. Arch Phys Med Rehabil 2002;83:329333.

33. Vassallo M, Vignaraja R, Sharma JC, et al. The effect of changing practice on fall prevention in a rehabilitative hospital: the Hospital Injury Prevention Study. J Am Geriatr Soc 2004;52:335-339.

34. Oliver D, Connelly JB, Victor CR, et al. Strategies to prevent falls and fractures in hospitals and care homes and effect of cognitive impairment: systematic review and meta-analyses. BMJ 2007;334:82.

\section{Management of transient ischaemic attack (TIA), stroke with spontaneous recovery, and minor non- disabling stroke \\ 11.1 Definition and causes of TIA}

- Transient ischaemic attack (TIA) is defined as a neurological deficit lasting $<24$ hours that is attributed to focal cerebral or retinal ischaemia. ${ }^{1}$

- The diagnosis is generally based on clinical history alone and specifically on the recollections and medical records of the patient who was neurologically impaired during the event.

- The initial aim of the requirement that resolution of symptoms occur within 24 hours was to exclude patients with infarction.

- However, infarctions can occur in those without persistent neurological deficits; evidence of acute infarction is identified by MRI in up to $50 \%$ of patients who meet the criteria for TIA. ${ }^{2}$ 
- About 15 to $20 \%$ of patients with stroke have a preceding TIA.

- The causes of TIA (such as atrial fibrillation (persistent or paroxysmal), carotid artery or vertebrobasilar artery disease (vessel to vessel embolisation or haemodynamic ischaemia) and large- and small-artery disease in the brain) are the same as those for stroke, so strategies to prevent further attacks are similar to those for stroke.

\subsection{Risk of stroke after TIA or minor non- disabling stroke}

- In a large North American study of patients presenting to the emergency units of 16 hospitals with physician-diagnosed TIA, $10.5 \%$ returned within 90 days of the index TIA with a stroke, but half of this risk is incurred within the first 2 days of the TIA. Of these strokes, $21 \%$ were fatal and $64 \%$ were disabling. ${ }^{4}$

- Other studies have confirmed a high risk of early stroke after TIA. ${ }^{5}$

- Patients with minor non-disabling stroke and rapid spontaneous clinical recovery are also at high risk of recurrent stroke or TIA. ${ }^{6,7}$

- The early risk of stroke is higher after posterior circulation territory events. $^{8}$

- The risk of stroke after non-retinal TIA attributable to a 70 - $99 \%$ stenosis of the internal carotid artery exceeded $25 \%$ within 3 months of the TIA.

\subsection{Evaluation of patients after TIA}

- TIA is often a neglected condition, and patients with TIA are frequently incorrectly managed without urgency and investigated 1 - 2 weeks later.

- TIA symptoms are often ignored by the patient or unrecognised by the doctor

- A detailed history is most helpful in determining potential causes of an episode of neurological impairment that appears consistent with a TIA.

- A full cardiovascular examination is an essential component of the evaluation of a patient with TIA. An ECG is recommended on all such patients, and further cardiac tests (e.g. echocardiography, Holter) may be required, depending on history and cardiovascular clinical examination.

- The neurological examination may identify persistent deficits that clarify the cause of the event.

- Simple clinical scoring systems can be used to identify patients at particularly high risk.

- $\mathrm{ABCD}^{2}$ score allows identification of groups at especially high risk in whom aggressive evaluation and urgent intervention is justified. ${ }^{10}$

- Laboratory tests (blood glucose, electrolytes, ESR, full blood count) may be useful in identifying TIA mimics (e.g. hypoglycaemia, hyponatraemia) or potential causes of the TIA (e.g. thrombocytosis, endocarditis, temporal arteritis).

- Carotid artery imaging is important to identify internal carotid artery stenosis as a cause of the TIA. Such imaging can be performed with Doppler ultrasonography, CT or MRI angiography. The latter two methods can also be used to image the vertebral arteries in the neck or the intracranial arteries, to identify a stenosis or dissection.

- CT brain scan or MRI may reveal evidence of acute infarction even if the patient has no symptoms or signs of stroke. Other mimics of TIA such as brain tumour or subdural haematoma may also be revealed.

\subsection{Treatment}

\subsubsection{Rapid assessment}

- Rapid assessment and intervention is the new standard for TIA care.

- Immediate preventive treatment ( see 11.4 .2 to 11.4 .8 below) will reduce stroke, disability and death. ${ }^{11,12}$

- Patient with TIA or minor non-disabling stroke require urgent clinical diagnosis to treat associated general abnormalities, modify active risk factors, identify specific treatable causes (particularly arterial stenosis and other embolic sources), and initiate secondary prevention to prevent stroke.

- Carotid artery imaging is a priority in those patients with TIA or minor stroke, more so than in those with major stroke where surgery is not going to be of benefit in the short term.

\subsubsection{Aspirin and other antiplatelet agents}

- Aspirin reduces the long-term risk of stroke and cardiovascular events after stroke or TIA with an overall reduction in risk of $22 \% .^{13}$

- Other antiplatelet drugs have not been tested specifically after TIA or as a treatment immediately after ischaemic stroke. In secondary prevention studies in patients with stroke, clopidogrel was slightly more effective than aspirin in reducing the risk of vascular events, and the combination of extended-release dipyridamole and aspirin was superior to aspirin alone in reducing the risk of stroke among patients who had previously had a stroke or TIA. ${ }^{14-16}$

\subsubsection{Anticoagulant therapy}

- Anticoagulation has not been evaluated specifically in patients with TIA but has been extensively evaluated after ischaemic stroke.

- In patients with stroke and atrial fibrillation, long-term oral anticoagulation reduces the risk of recurrent stroke. ${ }^{17}$

- Oral anticoagulation after non-cardiac ischaemic stroke is not superior to aspirin, and causes more bleeding. ${ }^{18-20}$

\subsubsection{Carotid endarterectomy (CEA)}

- CEA performed soon after TIA or minor stroke (see below) reduces the risk of recurrent disabling stroke or death (RR 0.52) in patients with severe (70 - 99\%) ipsilateral internal carotid artery stenosis (grading according to NASCET criteria). ${ }^{21-24}$

- CEA should only be performed in centres with a perioperative complication rate (all strokes and death) $<6 \%$.

- Men with less severe ipsilateral carotid stenosis (50 - 69\%) and very recent hemispheric symptoms may also benefit, provided the centre has a perioperative complication rate (all strokes and death) $<3 \% .^{24}$

- Women with severe ( $>70 \%$ ) symptomatic stenosis should undergo CEA, whereas women with more moderate stenosis should be treated medically. ${ }^{25}$

- Surgery is potentially harmful in patients with mild or moderate degrees of stenosis $(<50 \%)$. $^{24}$

- Older patients ( $>75$ years) without organ failure or serious cardiac dysfunction benefit from CEA. ${ }^{26}$

- Patients with amaurosis fugax, severe stenosis and a high risk profile should be considered for CEA; those with amaurosis fugax and few risk factors do better with medical treatment.

- Patients with mild-to-moderate intracranial stenosis and severe extracranial stenosis should be considered for CEA.

- The benefit from CEA is less in patients with lacunar stroke. ${ }^{27}$

- Patients with leukoaraiosis carry an increased perioperative risk. ${ }^{28}$

- Occlusion of the contralateral internal carotid artery (ICA) is not a contraindication to CEA but carries a higher perioperative risk. 
1 1.4.5 Timing of surgery for carotid stenosis after TIA or minor stroke

- The benefit from surgery is greatest in patients randomised within 2 weeks after the last ischaemic event, and falls rapidly with increasing delay (numbers needed to treat $=5$ within 2 weeks and falls to 125 after 12 weeks to prevent 1 ipsilateral stroke in 5 years). ${ }^{26}$

- No difference in operative complications observed between early ( $<3-6$ weeks) and late ( $>3-6$ weeks) surgery in stable patients.

\section{1.4.6 Carotid angioplasty and stenting (CAS)}

Several trials have compared CAS and CEA in secondary stroke prevention. ${ }^{29-32}$ An updated meta-analysis of these studies revealed a significantly higher risk of any stroke and death within 30 days after CAS, compared with CEA (OR 1.41; 95\% CI 1.07 - 1.87; $p=0.016)$. However, significant heterogeneity was found in this analysis $(p=0.035){ }^{33}$ After the periprocedural period, few ipsilateral strokes occurred with either procedure.

\subsubsection{Intracranial and vertebral artery occlusive disease}

- Extracranial-intracranial anastomosis between the superficial temporal and middle cerebral arteries is not beneficial in preventing stroke in patients with MCA or ICA stenosis or occlusion. ${ }^{34}$

- Patients with symptomatic intracranial stenoses $\geq 50 \%$ are at high risk of recurrent strokes, both in the anterior and posterior circulation (12\% after 1 year and $15 \%$ after 2 years in the territory of the stenosed artery). ${ }^{35,36}$

- Severe stenoses $(\geq 70 \%)$ carry a higher risk than moderate stenoses $(>50,<70 \%)^{36}$

- After stenting, recurrent strokes are reported in about $5-7 \%$ of patients with moderate or severe stenoses after 1 year, and in around $8 \%$ after 2 years. However, the incidence of complications after either angioplasty or stenting may be up to $6 \% .{ }^{37-41}$

- No RCTs have evaluated angioplasty, stenting or both for intracranial stenosis. Several non-randomised trials have shown feasibility and acceptable safety of intracranial stenting, but the risk of re-stenosis remains high. ${ }^{41,42}$

- Stenting of the extracranial segments of the vertebral artery is technically feasible with a moderate periprocedural risk as for example demonstrated in the SSYLVIA trial; but especially at the origin there is a particularly high rate of re-stenosis. ${ }^{42}$

- Endovascular procedures should only be undertaken in specialised comprehensive stroke centres that have experienced stroke

Risk stratification: $\mathrm{ABCD}_{2}$ score:

\begin{tabular}{|c|c|}
\hline Age: & $<60=0 ;>60=1$ \\
\hline \multirow[t]{2}{*}{ BP: } & Systolic BP $<140$ and/or diastolic BP $<90=0$ \\
\hline & Systolic BP $>140$ and/or diastolic BP $>90=1$ \\
\hline \multirow[t]{2}{*}{ Clinical: } & unilateral weakness $=2$ \\
\hline & $\begin{array}{l}\text { speech disturbance without weakness }=1 \\
\text { other symptoms }=0\end{array}$ \\
\hline \multirow[t]{3}{*}{ Duration: } & $<10 \min =0$ \\
\hline & $10-59 \min =1$ \\
\hline & $>59 \min =2$ \\
\hline Diabetes: & present $=1$ \\
\hline Scores 0 - & 3: low risk (risk of stroke within 2 days of TIA: 1\%) \\
\hline Scores 4 - & 5: moderate risk (risk of stroke within 2 days of TIA: $4.1 \%$ ) \\
\hline Scores 6 - ? & 7: high risk (risk of stroke within 2 days of TIA: 8.1\%) \\
\hline
\end{tabular}

neurologists or physicians (who are aware of risks/benefits) working with a highly skilled and properly trained neuro-interventionalist.

\subsubsection{Treatment of risk factors for cardiovascular disease}

Other medical interventions that reduce the risk of stroke among patients with a history of stroke or coronary artery or PVD are also likely to reduce the risk of vascular events after TIA (see Chapter 12, Secondary prevention)

\section{Recommendations}

- Patients with suspected TIA, or minor stroke with early spontaneous recovery, should be evaluated as soon as possible after an event (Class I, Level A).

- In patients with suspected TIA or stroke, urgent cranial CT (Class I) or MRI (Class II), is recommended within 24 hours of symptom onset (Level A).

- If MRI is used, the inclusion of diffusion-weighted imaging (DWI) and $\mathrm{T} 2$-weighted gradient echo sequences is recommended (Class II, Level A).

- Non-invasive imaging of the cervicocephalic vessels should be performed urgently and routinely as part of the evaluation of patients with suspected TIA (Class I, Level A).

- Non-invasive testing of the intracranial vasculature reliably excludes the presence of intracranial stenosis (Class I, Level A) and is reasonable to obtain when knowledge of intracranial stenoocclusive disease will alter management.

- ECG should be done as soon as possible after a TIA (Class I, Level B). Prolonged cardiac monitoring (inpatient telemetry or Holter monitor) is useful in patients with an unclear origin after initial brain imaging and echocardiography (Class II, Level B).

- Echocardiography is reasonable in the evaluation of patients with suspected TIAs, especially in patients in whom no cause has been identified by other elements of the workup (Class II, Level B).

- Routine blood tests (complete blood count, ESR, blood glucose and fasting lipids) are reasonable in the evaluation of patients with suspected TIAs (Class II, Level B).

- CEA is recommended for patients with $70-99 \%$ stenosis (Class I, Level A). CEA should only be performed in centres with a perioperative complication rate (all strokes and death) $<6 \%$ (Class I, Level A).

- CEA should be performed as soon as possible after the last ischaemic event, ideally within 2 weeks (Class II, Level B).

- CEA may be indicated for certain patients with stenosis of 50 $69 \%$; males with very recent hemispheric symptoms are most likely to benefit (Class III, Level C). CEA for stenosis of 50 - 69\% should only be performed in centres with a perioperative complication rate (all stroke and death) $<3 \%$ (Class I, Level A).

- CEA is not recommended for patients with stenosis $<50 \%$ (Class I, Level A).

- Patients should remain on antiplatelet therapy both before and after surgery (Class I, Level A).

- Carotid percutaneous transluminal angioplasty and/or stenting (CAS) is only recommended in selected patients (Class I, Level A). It should be restricted to the following subgroups of patients with severe symptomatic carotid artery stenosis: those with contraindications to CEA, stenosis at a surgically inaccessible site, re-stenosis after earlier CEA, and post-radiation stenosis (Class IV, GCP). Patients should receive a combination of clopidogrel and aspirin immediately before and for at least 1 month after stenting (Class IV, GCP). 
- Endovascular revascularisation by intravascular balloon angioplasty and/or stenting may be considered for patients with symptomatic severe intracranial stenoses (70\% luminal narrowing) despite optimal medical therapy (Class IV, GPC). Such procedures should be undertaken in specialised stroke centres.

References

1. Johnston SC. Transient ischaemic attack. N Engl J Med 2002;347(21):1687-1692.

2. Kidwell CS, Alger JR, Di Salie F, et al. Diffusion MRI in patients with transient ischemic attacks. Stroke 1999;30:1174-1180.

3. Rothwell PM, Warlow CP. Timing of TIA's preceding stroke: time window for prevention is very short. Neurology 2005:64:817-820.

4. Johnston SC, Gress DR, Browner WS, Sidney S. Short term prognosis after emergency department diagnosis of TIA. JAMA 2000;284:2901-2906.

5. Rothwell P, Buchan A, Johnston S. Recent advances in management of transient ischaemic attacks and minor ischaemic strokes. Lancet Neurol 2006;5:323-331.

6. Barber PA, Zhang J, Demchuk AM, Hill MD, Buchan AM. Why are stroke patients excluded from TPA therapy? An analysis of patient eligibility. Neurology 2001;56:1015-1020.

7. Prabhakaran S, Chong JY, Sacco RL. Impact of abnormal diffusion-weighted imaging results on shortterm outcome following transient ischemic attack. Arch Neurol 2007;64(8):1105-1109.

8. Flossman E, Rothwell PM. Prognosis of vertebrobasilar TIA and minor ischaemic stroke. Brain 2003;126:1940-1954

9. Streifler JY, Eliasziw M, Benavente OR, et al.The risk of stroke in patients with first-ever retinal vs hemispheric transient ischemic attacks and high-grade carotid stenosis. North American Symptomatic hemispheric transient ischemic attacks and high-grade carotid

10. Johnston SC, Rothwell PM, Nguyen-Huynh MN, et al. Validation and refinement of scores to predict very early stroke risk after transient ischaemic attack. Lancet 2007; 369:283-292.

11. Rothwell PM, Giles MF, Chandratheva A, et al. Effect of urgent treatment of transient ischaemic attack and minor stroke on early recurrent stroke (EXPRESS study): a prospective population-based sequential comparison. Lancet 2007;370:1432-1442.

12. Daffertshofer M, Mielke O, Pullwitt A, Felsenstein M, Hennerici M. Transient ischemic attacks are more than 'ministrokes'. Stroke 2004;35:2453-2458.

13. Antithrombotic Trialists' Collaboration. Collaborative meta-analysis of randomised trials of antiplatelet therapy for prevention of death, myocardial infarction, and stroke in high risk patients. BMJ 2002;324:71-86,141.

14. CAPRIE Steering Committee. A randomised, blinded, trial of clopidogrel versus aspirin in patients at risk of ischaemic events (CAPRIE). Lancet 1996;348:1329-1339.

15. Diener HC, Cunha L, Forbes C, Sivenius J, Smets P, Lowenthal A. European Stroke Prevention Study 2. Dipyridamole and acetylsalicylic acid in the secondary prevention of stroke. J Neurol Sci 1996;143:113.

16. Halkes PH, van Gijn J, Kappelle LJ, Koudstaal PJ, Algra A. Aspirin plus dipyridamole versus aspirin alone after cerebral ischaemia of arterial origin (ESPRIT): randomised controlled trial. Lancet 2006;367:1665-1673.

17. Ezekowitz MD. Levine JA. Preventing stroke in patients with atrial fibrillation. JAMA 1999;281:18301835.

18. Mohr JP, Thompson JL, Lazar RM, et al. A comparison of warfarin and aspirin for the prevention of recurrent ischemic stroke. N Engl J Med 2001;345:1444-1451.

19. The Stroke Prevention in Reversible Ischemia Trial (SPIRIT) Study Group: A randomized trial of anticoagulants versus aspirin after cerebral ischemia of presumed arterial origin. Ann Neurol $1997 ; 42: 857-865$

20. Algra A. Medium intensity oral anticoagulants versus aspirin after cerebral ischaemia of arterial origin (ESPRIT): a randomised controlled trial. Lancet Neurol 2007;6:115-124.

21. North American Symptomatic Carotid Endarterectomy Trial Collaborators: Beneficial effect of carotid endarterectomy in symptomatic patients with high-grade carotid stenosis. N Engl J Med 1991;325:445453.

22. Rothwell PM, Eliasziw M, Gutnikov SA, et al. Analysis of pooled data from the randomised controlled trials of endarterectomy for symptomatic carotid stenosis. Lancet 2003;361:107-116.

23. European Carotid Surgery Trialists' Collaborative Group: Endarterectomy for moderate symptomatic carotid stenosis: Interim results from the MRC European carotid surgery trial. Lancet 1996;347:1591 1593.

24. Cina C, Clase C, Haynes R. Carotid endarterectomy for symptomatic carotid stenosis. Cochrane Database of Systematic Reviews 1999

25. Rothwell PM, Eliasziw M, Gutnikov SA, Warlow CP, Barnett HJ. Sex difference in the effect of time from symptoms to surgery on benefit from carotid endarterectomy for transient ischemic attack and nondisabling stroke. Stroke 2004;35:2855-2861.

26. Rothwell PM, Eliasziw M, Gutnikov SA, Warlow CP, Barnett HJ. Endarterectomy for symptomatic carotid stenosis in relation to clinical subgroups and timing of surgery. Lancet 2004;363:915-924.

27. Inzitari D, Eliasziw M, Sharpe BL, Fox AJ, Barnett HJ. Risk factors and outcome of patients with carotid artery stenosis presenting with lacunar stroke. North American Symptomatic Carotid Endarterectomy Trial Group. Neurology 2000;54:660-666.

28. Streifler JY, Eliasziw M, Benavente OR, et al. Prognostic importance of leukoaraiosis in patients with symptomatic internal carotid artery stenosis. Stroke 2002;33:1651-1655.

29. Mas JL, Chatellier G, Beyssen B, et al., for the EVA-3S Investigators: Endarterectomy versus Stenting in Patients with Symptomatic Severe Carotid Stenosis. N Engl J Med 2006;355:1660-1671.

30. Ringleb PA, Allenberg JR, Berger J, et al. 30 day results from the SPACE trial of stent-protected angioplasty versus carotid endarterectomy in symptomatic patients: a randomised non-inferiority trial. Lancet 2006;368:1239-1247.

31. Yadav JS, Wholey MH, Kuntz RE, et al. Protected carotid-artery stenting versus endarterectomy in high-risk patients. N Engl J Med 2004;351:1493-1501.

32. Cavatas Group: Endovascular versus surgical treatment in patients with carotid stenosis in the Carotid and Vertebral Artery Transluminal Angioplasty Study (CAVATAS): a randomised trial. Lancet 2001;357:1729-1737.

33. Kastrup A, Groschel K. Carotid endarterectomy versus carotid stenting: an updated review of randomized trials and subgroup analyses. Acta Chir Belg 2007;107:119-128.
34. The EC/IC Bypass Study Group: Failure of extracranial-intracranial arterial bypass to reduce the risk of ischemic stroke. Results of an international randomized trial. N Engl J Med 1985;313:1191-1200.

35. Chimowitz MI, Lynn MJ, Howlett-Smith H, et al. Comparison of warfarin and aspirin for symptomatic intracranial arterial stenosis. N Engl J Med 2005;352:1305-1316.

36. Kasner SE, Chimowitz MI, Lynn MJ, et al. Predictors of ischemic stroke in the territory of a symptomatic intracranial arterial stenosis. Circulation 2006;113:555-563.

37. Jiang WJ, Xu XT, Du B, et al. Long-term outcome of elective stenting for symptomatic intracranial vertebrobasilar stenosis. Neurology 2007;68:856-858.

38. Jiang WJ, Xu XT, Du B, et al. Comparison of elective stenting of severe vs moderate intracranial atherosclerotic stenosis. Neurology 2007;68:420-426.

39. Marks MP, Wojak JC, Al-Ali F, et al. Angioplasty for symptomatic intracranial stenosis: clinical outcome. Stroke 2006;37:1016-1020

40. Fiorella D, Levy EI, Turk AS, et al. US multicenter experience with the wingspan stent system for the treatment of intracranial atheromatous disease: periprocedural results. Stroke 2007;38:881-887.

41. Bose A, Hartmann M, Henkes $\mathrm{H}$, et al. A novel, self-expanding, nitinol stent in medically refractory intracranial atherosclerotic stenoses: the Wingspan study. Stroke 2007;38:1531-1537.

42. SSYLVIA Study investigators: Stenting of Symptomatic Atherosclerotic Lesions in the Vertebral or Intracranial Arteries (SSYLVIA): study results. Stroke 2004;35:1388-1392.

\section{Secondary prevention}

Survivors of a transient ischemic attack (TIA) or stroke have an increased risk of another stroke, which is a major source of increased mortality and morbidity. Epidemiological studies have helped to identify the risk and determinants of recurrent stroke, and clinical trials have provided data to generate evidence-based recommendations to reduce this risk.

\subsection{Blood pressure management}

- Patients past the acute phase following a TIA or stroke benefit from BP-lowering medication, provided they are not suffering from symptomatic hypotension. ${ }^{1,2}$ The choice of the specific regimen has to be individualised and should follow the South African hypertension guideline. ${ }^{3}$

- However, BP should not be lowered intensively in patients with suspected haemodynamic stroke or in those with bilateral carotid stenosis.

- BP should be lowered and monitored indefinitely after stroke or TIA.

\subsection{Management of diabetes mellitus}

Near normoglycaemic glucose control leads to a reduction of vascular (including cerebrovascular) events in diabetic patients. ${ }^{4,5}$

\subsection{Management of hyperlipidaemia}

- HMG-CoA reductase inhibitors (statins) reduce the risk for subsequent cerebrovascular and cardiovascular events after TIA or stroke. ${ }^{6}$

- Patients with manifest vascular disease or at high risk for vascular events, with non-fasting total cholesterol levels $>3.5 \mathrm{mmol} / \mathrm{l}$, have fewer vascular events (including stroke) when treated with a statin, even when fasting total cholesterol levels are $<5.0 \mathrm{mmol} / \mathrm{l}$ and LDL cholesterol levels $<3.5 \mathrm{mmol} / \mathrm{l}^{7}$

- The risk of haemorrhagic stroke was slightly increased in both trials. ${ }^{6,7}$

- The absolute risk reduction achieved with statin therapy is low (NNT 112 - 143 for 1 year)

\subsection{Smoking cessation}

Cigarette smoking and exposure to environmental tobacco smoke are risk factors for ischaemic stroke. ${ }^{8,9}$ There are no specific data on secondary prevention.

\subsection{Alcohol moderation}

Alcohol consumption dose dependently increases the risk for stroke. Heavy drinkers are at higher risk compared with moderate drinkers. ${ }^{10}$ Men consuming $\leq 2$ units of alcohol daily and women consuming $\leq 1$ unit daily have, however, a mildly lower risk of stroke compared with 
persons consuming no alcohol at all. There are no specific data on secondary prevention.

\subsection{Weight reduction}

A BMI $>25$ and abdominal obesity (defined by waist circumference $>102 \mathrm{~cm}$ in men and $>88 \mathrm{~cm}$ in women) are associated with an increased risk for ischaemic stroke. ${ }^{11,12}$

\subsection{Physical activity}

Physical activity reduces the risk for stroke but there are no specific data on secondary prevention. ${ }^{13}$

\subsection{Platelet-inhibiting (antithrombotic) \\ medication}

- Aspirin reduces the risk for a subsequent cerebrovascular event after ischaemic stroke or TIA. ${ }^{14,15}$

- Clopidogrel is marginally more effective than aspirin in secondary stroke prevention. ${ }^{16}$

- The benefit of clopidogrel over aspirin is amplified in diabetics and patients with recurrent ischaemic strokes and myocardial infarctions. ${ }^{17,18}$

- The combination of extended release dipyridamole plus aspirin offers a small additional benefit over aspirin alone in reducing the chance of subsequent stroke. ${ }^{19,20}$

\subsection{Anticoagulation}

- Oral anticoagulation with warfarin is superior to aspirin in secondary stroke prevention for patients with non-valvular or valvular atrial fibrillation (AF), provided that intracranial haemorrhage has been excluded and the treatment can be properly supervised. The optimal INR for secondary stroke prevention in patients with AF is between 2.0 and 3.0. ${ }^{21-23}$

- Selected patients with proven cardio-embolic stroke benefit from anticoagulation with warfarin. ${ }^{24}$

- Anticoagulation with warfarin (INR 2.5 - 3.5) is superior to aspirin-based platelet-inhibiting drug regimens in the prevention of stroke in patients with mechanical prosthetic heart valves. ${ }^{25}$

- Oral anticoagulation is not recommended in patients with co-morbid conditions such as falls, poor compliance, uncontrolled epilepsy and gastro-intestinal bleeding.

- Increasing age alone is not a contra-indication to oral anticoagulation.

\subsection{Vascular interventions}

See paragraphs 11.4 .4 to 11.4 .6 .

\subsection{Patent foramen ovale (PFO)}

- Case reports and case control studies indicate an association between the presence of PFO and cryptogenic stroke in both younger and older stroke patients. ${ }^{26,27}$

- Two population-based studies pointed in the same direction but did not confirm a significant association. ${ }^{28,29}$

- In patients with PFO alone, the overall risk of recurrence is low. However, when PFO is combined with an atrial septal aneurysm, a eustachian valve or a Chiari network, or in patients who have suffered more than one stroke, the risk of recurrence can be substantial. $^{30}$

- Endovascular closure of PFOs with or without septal aneurysms is feasible in such patients and may lower the risk of recurrent stroke compared with medical treatment; however, RCTs are still lacking. ${ }^{31,32}$

\subsection{Sleep-disordered breathing}

- Sleep-disordered breathing is a risk factor and a consequence of stroke and has been linked with poorer long-term outcome and increased long-term stroke mortality. ${ }^{33}$

- Stroke patients may have sleep-disordered breathing, mostly in the form of obstructive sleep apnoea (OSA). This can improve spontaneously after stroke, but may need treatment. Continuous positive airway pressure is the treatment of choice for OSA.

\subsection{Postmenopausal oestrogen replacement therapy}

Hormone replacement therapy does not protect against vascular events and may increase stroke severity. 34

\section{Recommendations}

- Tight control of blood pressure $<130 / 80 \mathrm{mmHg}$ is recommended for secondary stroke prevention (Class I, Level A).

- Diabetic patients should be managed with lifestyle modification and individualised pharmacological therapy aiming for near normoglycaemic values. (Class IV, GCP).

- Patients with atherosclerotic stroke (non-cardio-embolic stroke) or TIA and non-fasting total cholesterol $>3.5 \mathrm{mmol} / \mathrm{l}$ should be treated with a statin (Class I, Level A).

- In the absence of documented efficacy of low-dosage statin treatment for secondary stroke prevention, the strength used in the secondary prevention trials should be prescribed (e.g. $40 \mathrm{mg}$ simvastatin).

- All health care providers should strongly advise patients with stroke or TIA to stop smoking and avoid environmental tobacco smoke (Class III, Level C).

- The heavy use of alcohol ( $>2$ drinks/day for men, and 1 drink/ day for non-pregnant women) should be discouraged (Class IV, GCP).

- Weight reduction is recommended for patients with a BMI $>25 \mathrm{~kg} /$ $\mathrm{m}^{2}$, and a waist circumference $>102 \mathrm{~cm}$ for men and $>88 \mathrm{~cm}$ for women (Class I Level B).

- Regular physical activity is recommended (Class IV, GCP). Patients with disability should be encouraged to engage in a supervised therapeutic exercise regimen.

- Patients with ischaemic stroke or TIA not requiring anticoagulation should receive platelet-inhibiting medication (Class I, Level A).

- Aspirin 75 - $150 \mathrm{mg} /$ day remains an inexpensive and cost-effective antiplatelet treatment for secondary prevention of stroke in a developing country.

- Alternatively, extended-release dipyridamole plus aspirin or clopidogrel can be considered (Class I, Level A), particularly in patients with recurrent vascular events, if already treated with aspirin, and in patients at high risk for cerebrovascular events.

- The combination of aspirin and clopidogrel is not recommended in patients with recent ischaemic stroke, except in patients with specific indications (e.g. unstable angina or non-Q-wave MI, or recent stenting) (Class I, Level A).

- Patients allergic to aspirin should receive clopidogrel.

- Anticoagulation with dose-adjusted warfarin is recommended for patients with cardio-embolic ischaemic stroke or TIA associated with intermittent or persistent AF (Class I, Level A).

- Patients with cardio-embolic stroke unrelated to AF should receive anticoagulants (INR 2.0 - 3.0) if the risk of recurrence is high (Class III, Level C).

- Patients unable to take oral anticoagulants should receive plateletinhibiting medication (Class IV, GCP). 
- For patients with cardio-embolic stroke or TIA following acute myocardial infarction, oral anticoagulation between 3 and 12 months is reasonable (Class IV, GCP).

- Endovascular closure of PFO may be considered in patients with cryptogenic stroke and high risk PFO (Class IV, GCP).

- Sleep-disordered breathing such as obstructive sleep apnoea should be treated with continuous positive airway pressure breathing (Class III, Level GCP).

- Hormone replacement therapy is not recommended for the secondary prevention of stroke (Class I, Level A).

\section{References}

1. PROGRESS Collaborative Group. Randomised trial of a perindopril-based blood-pressure-lowering regimen among 6105 individuals with previous stroke or transient ischaemic attack. Lancet 2001;358:1033-1041.

2. Rashid P, Leonardi-Bee J, Bath P. Blood pressure reduction in secondary prevention of stroke and other vascular events: a systematic review. Stroke 2003;34:2741-2748.

3. Seedat YK, Croasdale MA, Milne FJ, et al. Guideline Committee, Southern African Hypertension Society; Directorate: Chronic Diseases, Disabilities and Geriatrics, National Department of Health. South African hypertension guideline 2006. S Afr Med J 2006;96(4 Pt 2):337-362.

4. American Diabetes Association. Standards of medical care for patients with diabetes mellitus. Diabetes Care 2003;26(suppl 1):S33-S50.

5. Intensive blood glucose control with sulphonylureas or insulin compared with conventional treatment and risk of complications in patients with type 2 diabetes (UKPDS 33): UK Prospective Diabetes Study (UKPDS) Group. Lancet 1998;352:352:837-853.

6. Amarenco P, Bogousslavsky J, Callahan A, et al. High-dose atorvastatin after stroke or transient ischemic attack. N Engl J Med 2006;355:549-559.

7. Heart Protection Study Collaborative Group: MRC/BHF heart protection study of cholesterol lowering with simvastatin in 20,536 high-risk individuals: a randomised placebo-controlled trial. Lancet with simvast $2002 ; 360: 7-22$

8. Shinton R, Beevers G. Meta-analysis of relation between cigarette smoking and stroke. BMJ 1989;298:789-794.

9. Ong MK, Glantz SA. Cardiovascular health and economic effects of smoke-free workplaces. Am J Med 2004;117:32-38.

10. Reynolds K, Lewis B, Nolen JD, Kinney GL, Sathya B, He J. Alcohol consumption and risk of stroke: a meta-analysis. JAMA 2003;289:579-588.

11. Kurth T, Gaziano JM, Berger K, et al. Body mass index and the risk of stroke in men. Arch Intern Med 2002;162:2557-2562

12. AHA/ACC Guidelines for secondary prevention for patients with coronary and other atherosclerotic vascular disease: 2006 update. J Am Coll Cardiol 2006;47:2130-2139.

13. Lee CD, Folsom AR, Blair SN. Physical activity and stroke risk: a meta-analysis. Stroke 2003;34:24752481

14. Antithrombotic Trialists' Collaboration. Collaborative meta-analysis of randomised trials of antiplatelet therapy for prevention of death, myocardial infarction, and stroke in high-risk patients. BMJ 2002;324:71-86.

15. Collaborative overview of randomised trials of antiplatelet therapy, I: prevention of death, myocardial infarction, and stroke by prolonged antiplatelet therapy in various categories of patients: Antiplatelet Trialists' Collaboration. BMJ 1994;308:81-106.

16. CAPRIE Steering Committee. A randomised, blinded, trial of clopidogrel versus aspirin in patients at risk of ischemic events (CAPRIE): CAPRIE steering committee. Lancet 1996;348:1329-1339.

17. Bhatt DL, Marso SP, Hirsch AT, Ringleb PA, Hacke W, Topol EJ. Amplified benefit of clopidogrel versus aspirin in patients with diabetes mellitus. Am J Cardiol 2002;90:625-628.

18. Ringleb PA, Bhatt DL, Hirsch AT, Topol EJ, Hacke W, for the clopidogrel versus aspirin in patients at risk of ischemic events investigators. Benefit of clopidogrel over aspirin is amplified in patients with a risk of ischemic events investigators. Benefit of clop
history of ischemic events. Stroke 2004;35:528-532.

19. Diener HC, Cunha L, Forbes C, Sivenius J, Smets P, Lowenthal A. European Stroke Prevention Study, 2: Dipyridamole and acetylsalicylic acid in the secondary prevention of stroke. J Neurol Sci 1996;143:113.

20. Halkes PH, van Gijn J, Kappelle LJ, Koudstaal LJ, Algra A. ESPRIT Study Group. Aspirin plus dipyridamole versus aspirin alone after cerebral ischaemia of arterial origin (ESPRIT): randomised controlled trial. Lancet 2006;367:1637-1665

21. Risk factors for stroke and efficacy of anti-thrombotic therapy in atrial fibrillation: analysis of pooled data from five randomised controlled trials. Arch Intern Med 1994;154:1449-1457.

22. Secondary prevention in non-rheumatic atrial fibrillation after transient ischaemic attack or minor stroke: EAFT (European Atrial Fibrillation Trial) Study Group. Lancet 1993;342:1255-1262.

23. Roy D, Marchand E, Gagne P, Chabot M, Cartier R. Usefulness of anticoagulant therapy in the prevention of embolic complications of atrial fibrillation. Am Heart J 1986;112:1039-1043.

24. Visser CA, Kan G, Meltzer RS, Lie KI, Durrer D. Long-term follow-up of left ventricular thrombus after acute myocardial infarction: a two-dimensional echocardiographic study in 96 patients. Chest 1984;86:532-536

25. Mok CK, Boey J, Wang R, et al. Warfarin versus dipyridamole-aspirin and pentoxifylline-aspirin for the prevention of prostheticheartvalvethromboembolism:aprospectiverandomizedclinicaltrial.Circulation 1985;72:1059-1063.

26. Handke M, Harloff A, Olschewski M, Hetzel A, Geibel A. Patent foramen ovale and cryptogenic stroke in older patients. N Engl J Med 2007;357:2262-2268.

27. Overell JR, Bone I, Lees KR. Interatrial septal abnormalities and stroke: a meta-analysis of case-control studies. Neurology 2000;55:1172-1179.

28. Di Tullio MR, Sacco RL, Sciacca RR, Jin Z, Homma S. Patent foramen ovale and the risk of ischemic stroke in a multiethnic population. J Am Coll Cardiol 2007;49:797-802.

29. Meissner I, Khandheria BK, Heit JA, et al. Patent foramen ovale: innocent or guilty? Evidence from a prospective population-based study. J Am Coll Cardiol 2006;47:440-445.

30. Mas JL, Arquizan C, Lamy C, et al. Recurrent cerebrovascular events associated with patent foramen ovale, atrial septal aneurysm, or both. N Engl J Med 2001;345:1740-1746.
31. Wahl A, Krumsdorf U, Meier B, et al. Transcatheter treatment of atrial septal aneurysm associated with patent foramen ovale for prevention of recurrent paradoxical embolism in high-risk patients. J Am patent foramen ovale for presion $2005 ; 45: 377-380$
Coll Cardiol

32. Windecker S, Wahl A, Nedeltchev K, et al. Comparison of medical treatment with percutaneous closure of patent foramen ovale in patients with cryptogenic stroke. J Am Coll Cardiol 2004;44:750-758.

33. Bassetti CL. Sleep and stroke. Semin Neurol 2005;25:19-32.

34. Viscoli CM, Brass LM, Kernan WN, Sarrel PM, Suissa S, Horwitz RI. A clinical trial of estrogenreplacement therapy after ischemic stroke. N Engl J Med 2001;345:1243-1249.

\section{Stroke rehabilitation}

\subsection{Definition}

- Stroke rehabilitation is a goal-orientated process which attempts to obtain maximum function in patients who have had strokes and who suffer from a combination of physical, cognitive and language disabilities.

- The rehabilitation process is best performed using an interdisciplinary approach by experts who have experience and understanding of the particular issues facing stroke patients. ${ }^{1}$

\subsection{Goals}

- The ultimate goal of rehabilitation is to enable patients to resume their pre-morbid function both within family and community life and, if possible, at work. Where this is not possible, alternative strategies should be considered.

- If full resumption of work activities is not possible, skills retraining may be considered.

- In patients who have significant permanent disabilities, consideration must be aimed at reducing the burden of care for the family and helping the patient to become as independent as possible.

- With patients who have had severe strokes with poor recovery, the provision and training of caregivers should be addressed by the rehabilitation team and, in very poor outcomes, institutionalisation may be recommended for the patient's ultimate care. Decisions about home care v. institutionalisation must involve all parties and should take into account financial and social circumstances.

- Rehabilitation can be organised on an in- or outpatient basis.

- In the South African context, outpatient therapy very rarely achieves the intensity of inpatient rehabilitation units. Therefore, where necessary, inpatient therapy may be preferable. Criteria for admission to inpatient therapy vary. Typically, where patients require three modalities of intervention, or where patients are unable to transfer independently, inpatient rehabilitation is justified; this is particularly true in patients with moderate and severe strokes.

- The majority of stroke patients in South Africa are treated in the public health care sector where there is currently a shortage or even absence of inpatient rehabilitation beds for stroke patients, especially in rural and remote areas. Under such circumstances, efforts are currently underway to improve home-based care and rehabilitation as well as community-based rehabilitation and the use of 'step-down' facilities where available.

\subsection{Interdisciplinary approach}

- A key characteristic of the stroke unit model of care is rehabilitation delivered by a specialised multidisciplinary team who communicate with each other regularly and use their varying expertise to work towards common goals. ${ }^{1,2}$

- Admission to a dedicated stroke unit improves outcomes for all strokes irrespective of age, sex and severity. ${ }^{3}$

- There are also long-term functional benefits of dedicated stroke unit care; follow-up at 5 and 10 years has revealed persisting efficacy compared with controls. ${ }^{4,5}$

- Early initiation of rehabilitation interventions has been associated with improved outcome at discharge from hospital and at follow-up. ${ }^{1}$ 
- The financial and social implications of prolonged hospitalisation have prompted increasing interest in services to facilitate early return to the community.

- A multidisciplinary team approach can significantly reduce bed days for selected stroke patients who have mild or moderate impairment at baseline, but mortality has been shown to increase when patients were discharged early with only generic community support. $^{6-8}$

- The rehabilitation team usually consists of the following personnel: physiotherapist, occupational therapist, speech and language therapist, psychologist, social worker, dietician, nurses who have training and experience in rehabilitation, and medical practitioner with an understanding of the stroke and rehabilitation processes.

- The patient should be fully assessed by each member or the team within 24 hours of the onset of the stroke. After the initial assessment, therapy strategies are planned and a decision for in- or outpatient therapy is suggested.

- An essential aspect of the interdisciplinary team approach is the issue of interaction and communication with both the patient and the family.

- Ideally, communication between the rehabilitation team, family and patient should be formalised at a family meeting within the first week of therapy. The aim of the family meeting is to educate the patient and family on the circumstances of the particular patient and the anticipated outcomes.

\subsection{Education}

Education of the patient, family members and caregivers is an important aspect of stroke rehabilitation, requiring participation by all members of the team. Issues that need to be discussed include causes of stroke and investigations, rehabilitation interventions, prevention of repeated strokes, outcomes after stroke, necessary lifestyle changes, sexuality, impact on family, resumption of driving, participation in leisure activities, and complications.

\subsection{Discharge planning}

- Discharge planning is an integral part of rehabilitation.

- At an early stage, provisional plans for discharge should be considered. These plans may change during the process of rehabilitation.

- Early planning will enable families to implement changes that may be necessary within the home, such as alterations to toilet and bath facilities and the construction of ramps, where necessary. Special consideration must be given to patients who are returning to informal settlements and shacks, with no access to basic amenities.

- In urban inpatient facilities, it is common practice to allow patients to go home for one or two nights prior to their discharge, so enabling the patient and the family to assess what difficulties are encountered in their home environment and to use the rehabilitation team to address these problems and correct them before final discharge. In a rural setting, a home visit by a member of the rehabilitation team prior to discharge may assist in planning a home adaptation programme. As far as possible, patients in rural areas should also have the opportunity to go home for one or two nights prior to discharge.

- Discharge planning should also include the possibility of returning to work and implementing changes in the work place which may be appropriate for the patient.

- At the time of discharge, appropriate referral to various health care professionals and support groups is important. This may include a delayed referral to an occupational therapist who specialises in performing work assessments.

- Liaison between the rehabilitation team and employers is also important, and is usually performed by the social worker.

- In patients where significant and permanent disability is anticipated, the concept of caregiver employment and training should be implemented timeously.

\subsection{Post-discharge rehabilitation}

- Rehabilitation is a protracted process and, once the patient is discharged from hospital or from the rehabilitation unit, ongoing therapy may be necessary. Appropriate referrals for therapy should be made before the time of discharge. A meta-analysis showed that continued rehabilitation after discharge during the first year after stroke reduces the risk of deterioration in function and improves daily living activities. ${ }^{9}$ The interventions included occupational therapy, physiotherapy, and multidisciplinary teams, and therefore no definitive statement can be made concerning the optimal mode of service delivery.

- It is common practice to re-assess patients some time after discharge from their rehabilitation programme to assess their progress and to address any new difficulties which have been identified by patients and their families.

\subsection{Rehabilitation interventions}

\subsubsection{Physiotherapy}

- Early intervention by physiotherapists will be concerned with appropriate positioning of hemiplegic patients and attempts at early mobilisation and mobility.

- Safety of the patient and the prevention of falls and injury are of paramount importance at all times.

- Communication between physiotherapist and nursing staff is important in maintaining levels of safety.

- There is no clearly superior model of physiotherapy for stroke rehabilitation, but some evidence exists to support specific interventions. ${ }^{10,11}$

- Several groups have shown that strength can be improved in a dose-dependent manner, without increasing spasticity. ${ }^{12}$

- The South African Society of Physiotherapy has produced a document detailing its recommended physiotherapy management of stroke patients ('CVA essential healthcare package').

\subsubsection{Occupational therapy (OT)}

- Initially, this concerns resumption of daily activities such as grooming, toileting, washing, shaving, dressing and eating.

- Issues of neglect, spatial perception problems and visual difficulties must be considered in the course of OT sessions.

- Assistive devices may be required; and it is the occupational therapist's role to identify which devices are required. These may include equipment such as wheelchairs, walking aids and bath and shower aids.

- A systematic review of 9 trials comparing occupational therapybased ADL therapy with usual care reported improved functional outcomes in the active intervention group. ${ }^{13}$ The data do not justify conclusions on the optimal mode of OT delivery.

- A meta-analysis of community-based OT trials found improved performance on ADL measures. The greatest effects were seen in older patients and with the use of targeted interventions. ${ }^{14}$

\subsubsection{Speech therapy}

- An important role of the speech and language therapist in early 
intervention is the assessment of swallowing safety. This is extremely important; failure to identify and implement appropriate measures for swallowing safety may result in significant increases in morbidity and mortality.

- In patients who have language difficulties, the involvement of speech and language therapists from the onset is important. Alternative communication techniques may be explored, and education of family members, particularly about the levels of frustration experienced by people who are aphasic, must be discussed.

\subsubsection{Diet}

Involvement of a dietician early in the recovery phase is important. Malnutrition has been shown to be a significant problem in patients with strokes, either because of difficulty with swallowing or the inability of patients to express their needs about nutritional requirements (aphasic patients).

\subsubsection{Psychological support}

- Stroke often has a devastating effect on patients and their families. The psychologist may have an important role in counselling the stroke patient and family members about adapting to disability, and new roles within the family and workplace.

- Depression is a common consequence of stroke. ${ }^{15}$ Particular issues which contribute to depression include fear of further strokes, loss of independence, loss of earning power, altered social dynamics and loss of function.

- Post-stroke depression is associated with poor rehabilitation results and ultimately poor outcome. ${ }^{16,17}$

- In clinical practice, only a minority of depressed patients are diagnosed, and even fewer are treated. ${ }^{18}$

- Predictors of post-stroke depression in the rehabilitation setting include increasing physical disability, cognitive impairment and stroke severity. ${ }^{17}$

- The routine use of antidepressants is not recommended.

- Where depression has been identified as a persistent problem, intervention by psychotherapists and the use of antidepressant medication should be considered.

- Antidepressant drugs such as selective serotonin reuptake inhibitors (SSRIs) and heterocyclics can improve mood after stroke, but there is less evidence that these agents can effect full remission of a major depressive episode or prevent depression. SSRIs are better tolerated than heterocyclics. ${ }^{19-21}$

- There is no good evidence to recommend psychotherapy for treatment or prevention of post-stroke depression, although such therapy can elevate mood. ${ }^{22}$

- Emotionalism is a distressing symptom for patients and carers. SSRIs may reduce emotional outbursts but effects on quality of life are not clear. ${ }^{23}$

\subsubsection{Counselling on sexuality}

- Sexuality can suffer after a stroke.

- Change in body image, underlying physical limitations, and co-morbid vascular disease may be compounded by side-effects of medications. ${ }^{24}$

- Issues of sexuality and intimacy should be discussed with patients, preferably by an appropriately trained professional. ${ }^{25}$

- Provision of support and information is important: many patients wrongly fear that resuming an active sex life may result in further stroke. ${ }^{26}$
13.8 Complications affecting rehabilitation

- Rehabilitation can be compromised by complications that may be strong predictors of poor functional outcome and mortality. Common complications during inpatient rehabilitation include depression, shoulder pain, thalamic (central) pain, spasticity, falls, urinary disturbances and aspiration pneumonia. ${ }^{27}$

- Post-stroke shoulder pain is common especially in patients with impaired arm function and poor functional status, and is associated with poorer outcome. ${ }^{28}$

- Passive movement of a paretic limb may be preventive. ${ }^{29}$

- Electrical stimulation is commonly used for treatment, but its efficacy is unproven. ${ }^{30}$ A Cochrane systematic review found insufficient data to recommend the use of orthotic devices for shoulder subluxation, despite a trend towards efficacy of arm strapping the affected limb. ${ }^{31}$

- Lamotrigine and gabapentin may be considered for neuropathic pain. ${ }^{32}$ They appear to be well tolerated, but cognitive side-effects should be considered.

- Spasticity in the chronic phase may adversely affect ADL and quality of life..$^{33}$ Posture and movement therapy, relaxing therapy, splints and supports are all commonly employed, but a sound evidence base is lacking. ${ }^{34}$ Pharmacotherapy with botulinum toxin has proven effects on muscle tone in arms and legs, but functional benefits are less well studied. ${ }^{35-37}$ Oral agents are limited in their use because of side-effects. ${ }^{38}$

\subsection{Eligibility for rehabilitation}

- In South Africa, access to and availability of either inpatient or outpatient rehabilitation varies considerably, as does the duration and intensity of the rehabilitation programme. This depends not only the severity of the neurological deficits but also on a number of other factors relevant to developing counties: whether or not the stroke patient has private health insurance (a minority of the country's stroke patients have), their geographical location, and availability of trained rehabilitation therapists and public or private rehabilitation facilities in the area (most are located in the larger metropolitan areas).

- An important predictor of rehabilitation outcome is initial stroke severity ${ }^{16}$ Pre-stroke disability is clearly also a strong determinant of outcome. ${ }^{39}$

- Exclusion from rehabilitation on the basis of pre-stroke dependence remains a contentious issue. ${ }^{40,41}$

- Limited facilities and resource constraints in South Africa frequently preclude patients with pre-stroke dependency or other reasons deemed to be prognostic of a very poor outcome from access to inpatient rehabilitation.

- Patients with the most severe cognitive or physical impairments have been excluded from most rehabilitation trials, and therefore caution is required in extrapolating results to this group. ${ }^{42}$

- For those unable to participate actively, passive movements to prevent contractures or pressure sores have been recommended. ${ }^{43}$

\subsection{Development of a model for stroke care in under-resourced settings}

- In-service training of health care professionals and homebased carers in all aspects of stroke care would be an important component in the future planning and implementation of both hospital and community-based stroke services in an underresourced setting such as South Africa.

- Any model of community-based stroke care in South African settings should include a system of stroke education for caregivers 
and patients, and should implement structures that strengthen the level of home-based care and training.

- Awareness of stroke and cardiovascular risk factors, particularly hypertension, needs to be fostered through improved community education. Nurse practitioners and home-based carers could play an important role in checking BP and monitoring treatment and compliance after discharge from hospital.

- In-service training of both urban and rural-based health care professionals in protocols of acute stroke management might reduce in-hospital complications, morbidity and mortality, providing optimal potential for an improved outcome following discharge into the community.

- In the absence of adequate numbers of health workers available for rehabilitation in such communities, caregivers are the likely candidates to adopt this surrogate role, and could be trained to be more active in the rehabilitation process. ${ }^{4}$

- These relatively simple interventions have the potential to improve stroke outcomes and relieve caregiver strain in the South African setting.

\section{Recommendations}

- Early initiation of rehabilitation is recommended (Class III, Level C).

- Early discharge from stroke unit care is possible in medically stable patients with mild or moderate impairment, providing that rehabilitation is delivered in the community by a multidisciplinary team with stroke expertise (Class I, Level A).

- Rehabilitation should be continued after discharge during the first year after stroke (Class II, Level A).

- Any model of community-based stroke care in South African settings should include a system of stroke education for caregivers and patients, and should implement structures that strengthen the level of home-based care and training (Class IV, GCP).

\section{Reference}

1. Cifu DX, Stewart DG. Factors affecting functional outcome after stroke. A critical review of rehabilitation intervention. Arch Phys Med Rehab 1999;80(5, suppl1):S35-39.

2. Langhorne P, Dennis MS. Stroke Units, an Evidence Based Approach. London: BMJ Publishing, 1998.

3. Stroke Unit Trialists' Collaboration: Organised inpatient (stroke unit) care for stroke. Cochrane Database Syst Rev 2007:CD000197.

4. Lincoln NB, Husbands S, Trescoli C, Drummond AE, Gladman JR, Berman P. Five-year follow-up of randomised controlled trial of a stroke rehabilitation unit. BMJ 2000;320:549.

Indredavik B, Slordahl SA, Bakke F, Rokseth R, Haheim LL. Stroke unit treatment. Long-term effects. Stroke 1997;28:1861-1866.

6. Early supported discharge trialists: Services for reducing duration of hospital care for acute stroke patients. Cochrane Database Syst Rev 2005:CD000443.

7. Langhorne P, Taylor G, Murray G, et al. Early supported discharge services for stroke patients: a metaanalysis of individual patients' data. Lancet 2005;365:501-506

8. Ronning OM, Guldvog B. Outcome of subacute stroke rehabilitation: a randomized controlled trial. Stroke 1998;29:779-784

9. Legg L, Langhorne P. Rehabilitation therapy services for stroke patients living at home: systematic review of randomised trials. Lancet 2004:363:352-356.

10. van Peppen RP, Kwakkel G, Wood-Dauphinee S, Hendriks HJ, Van der Wees PJ, Dekker J. The impact of physical therapy on functional outcomes after stroke: what's the evidence? Clin Rehabil 2004;18:833862.

11. Pollock A, Baer G, Langhorne P, Pomeroy V. Physiotherapy treatment approaches for the recover of postural control and lower limb function following stroke: a systematic review. Clin Rehabil 2007;21:395-410.
12. van der Lee JH, Snels IA, Beckerman H, Lankhorst GJ, Wagenaar RC, Bouter LM. Exercise therapy for arm function in stroke patients: a systematic review of randomized controlled trials. Clin Rehabil 2001;15:20-31.

13. Legg LA, Drummond AE, Langhorne P. Occupational therapy for patients with problems in activities of daily living after stroke. Cochrane Database Syst Rev 2006:CD003585.

4. Walker MF, Leonardi-Bee J, Bath P, et al. Individual patient data meta-analysis of randomized controlled trials of community occupational therapy for stroke patients. Stroke 2004;35:2226-2232.

15. Linden T, Blomstrand C, Skoog I. Depressive disorders after 20 months in elderly stroke patients: a case-control study. Stroke 2007;38:1860-1863.

16. Paolucci S, Antonucci G, Pratesi L, Traballesi M, Lubich S, Grasso MG. Functional outcome in stroke inpatient rehabilitation: predicting no, low and high response patients. Cerebrovasc Dis 1998;8:228234

17. Hackett ML, Anderson CS. Predictors of depression after stroke: a systematic review of observational studies. Stroke 2005;36:2296-2301

18. Paolucci S, Gandolfo C, Provinciali L, Torta R, Toso V. The Italian multicenter observational study on post-stroke depression (DESTRO). J Neurol 2006;253:556-562.

19. van de Meent H, Geurts AC, Van Limbeek J. Pharmacologic treatment of poststroke depression: a systematic review of the literature. Top Stroke Rehabil 2003;10:79-92.

20. Hackett ML, Anderson CS, House AO. Management of depression after stroke: a systematic review of pharmacological therapies. Stroke 2005;36:1098-1103.

21. Bhogal SK, Teasell R, Foley N, Speechley M. Heterocyclics and selective serotonin reuptake inhibitors in the treatment and prevention of poststroke depression. J Am Geriatr Soc 2005;53:1051-1057.

22. Anderson CS, Hackett ML, House AO. Interventions for preventing depression after stroke. Cochrane Database Syst Rev 2004:CD003689.

23. House AO, Hackett ML, Anderson CS, Horrocks JA. Pharmaceutical interventions for emotionalism after stroke. Cochrane Database Syst Rev 2004:CD003690.

24. Marinkovic S, Badlani G. Voiding and sexual dysfunction after cerebrovascular accidents. J Urol 2001:165:359-370.

25. Sjogren K, Fugl-Meyer AR. Adjustment to life after stroke with special reference to sexual intercourse and leisure. J Psychosom Res 1982;26:409-417.

26. Muller JE. Triggering of cardiac events by sexual activity: findings from a case-crossover analysis. Am J Cardiol 2000;86:14F-18F

27. McLean DE. Medical complications experienced by a cohort of stroke survivors during inpatient, tertiary-level stroke rehabilitation. Arch Phys Med Rehabil 2004;85:466-469.

28. Lindgren I, Jonsson AC, Norrving B, Lindgren A. Shoulder pain after stroke: a prospective populationbased study. Stroke 2007;38:343-348.

29. Vuagnat $\mathrm{H}$, Chantraine A. Shoulder pain in hemiplegia revisited: contribution of functional electrical stimulation and other therapies. J Rehabil Med 2003;35:49-54.

30. Price CI, Pandyan AD. Electrical stimulation for preventing and treating post-stroke shoulder pain: a systematic Cochrane review. Clin Rehabil 2001;15:5-19.

1. Ada L, Foongchomcheay A, Canning C. Supportive devices for preventing and treating subluxation of the shoulder after stroke. Cochrane Database Syst Rev 2005:CD003863.

32. Wiffen P, Collins S, McQuay H, Carroll D, Jadad A, Moore A. Anticonvulsant drugs for acute and chronic pain. Cochrane Database Syst Rev 2005:CD001133.

33. Satkunam LE. Rehabilitation medicine: 3 . Management of adult spasticity. CMAJ 2003;169:1173-1179.

34. Lannin NA, Herbert RD. Is hand splinting effective for adults following stroke? A systematic review and methodologic critique of published research. Clin Rehabil 2003;17:807-816.

35. Brashear A, Gordon MF, Elovic E, et al. Intramuscular injection of botulinum toxin for the treatment of wrist and finger spasticity after a stroke. N Engl J Med 2002;347:395-400.

36. van Kuijk AA, Geurts AC, Bevaart BJ, van Limbeek J. Treatment of upper extremity spasticity in stroke patients by focal neuronal or neuromuscular blockade: a systematic review of the literature. J Rehabil Med 2002;34:51-61.

37. Pittock SJ, Moore AP, Hardiman O. A double-blind randomised placebo-controlled evaluation of three doses of botulinum toxin type A (Dysport) in the treatment of spastic equinovarus deformity after stroke. Cerebrovasc Dis 2003;15:289-300.

38. Meythaler JM, Guin-Renfroe S, Johnson A, Brunner RM. Prospective assessment of tizanidine for spasticity due to acquired brain injury. Arch Phys Med Rehabil 2001:82:1155-1163.

39. Shah S, Vanclay F, Cooper B. Efficiency, effectiveness, and duration of stroke rehabilitation. Stroke 1990;21:241-246

40. Gladman JR, Sackley CM. The scope for rehabilitation in severely disabled stroke patients. Disabil Rehabil 1998;20:391-394.

41. Rodgers H. The scope for rehabilitation in severely disabled stroke patients. Disabil Rehabil 2000;22:199200.

42. van Peppen RP, Hendriks HJ, van Meeteren NL, Helders PJ, Kwakkel G. The development of a clinical practice stroke guideline for physiotherapists in The Netherlands: a systematic review of available evidence. Disabil Rehabil 2007;29:767-783.

43. The European Stroke Initiative Executive Committee and the EUSI Writing Committee. European Stroke Initiative Recommendations for Stroke Management - Update 2003. Cerebrovasc Dis 2003; 16:311-337.

44. Wasserman S, de Villiers L, Bryer A. Community-based care of stroke patients in a rural African setting. S Afr Med J 2005:95(3):630-635.

Accepted 3 August 2010. 